Research Article

\title{
Bifurcation and Chaos of a Discrete Predator-Prey Model with Crowley-Martin Functional Response Incorporating Proportional Prey Refuge
}

\author{
P. K. Santra, ${ }^{1}$ G. S. Mahapatra, ${ }^{2}$ and G. R. Phaijoo ${ }^{3}{ }^{3}$ \\ ${ }^{1}$ Maulana Abul Kalam Azad University of Technology, Kolkata 700064, India \\ ${ }^{2}$ Department of Mathematics, National Institute of Technology Puducherry, Karaikal 609609, India \\ ${ }^{3}$ Department of Mathematics, Kathmandu University, Dhulikhel 45200, Nepal
}

Correspondence should be addressed to G. R. Phaijoo; gangaram@ku.edu.np

Received 12 September 2019; Revised 29 November 2019; Accepted 6 December 2019; Published 22 January 2020

Academic Editor: Elisa Francomano

Copyright (c) 2020 P. K. Santra et al. This is an open access article distributed under the Creative Commons Attribution License, which permits unrestricted use, distribution, and reproduction in any medium, provided the original work is properly cited.

The paper investigates the dynamical behaviors of a two-species discrete predator-prey system with Crowley-Martin functional response incorporating prey refuge proportional to prey density. The existence of equilibrium points, stability of three fixed points, period-doubling bifurcation, Neimark-Sacker bifurcation, Marottos chaos, and Control Chaos are analyzed for the discrete-time domain. The time graphs, phase portraits, and bifurcation diagrams are obtained for different parameters of the model. Numerical simulations and graphics show that the discrete model exhibits rich dynamics, which also present that the system is a chaotic and complex one. This paper attempts to present a feedback control method which can stabilize chaotic orbits at an unstable equilibrium point.

\section{Introduction}

The dynamical behavior of the prey-predator system in ecology has been the subject of study for many researchers and hence they have provided a substantial contribution to the growth of the population models [1-8] in the continuous-time domain. In case of populations that have overlapping generations and the birth processes occuring continuously, the ordinary differential equations are used for modeling of predator-prey interaction [9-30]. Many species, such as monocarpic plants, and semelparous animals have discrete non overlapping generations, and their births occur in regular breeding seasons. Their interactions are characterized by difference equations or formulated as discretetime mappings. Discrete-time predator-prey models are capable of exhibiting much more complicated dynamics in comparison to the corresponding continuous-time models [31-45].

The dynamical study of the prey-predator model with different functional responses is an effective approach for time domain. Upadhyay and Naji [46] presented threespecies food chain model with Crowley-Martin type functional response for the dynamical study. Zhou [47] presented qualitative analysis of the predator-prey model for Crowley-Martin functional responses. Tripathi et al. [48] applied Crowley-Martin functional response on a delayed density dependent predator-prey model. Li et al. [49] presented a predator-prey model with Crowley-Martin functional response for positive periodic solutions.

A certain degree of protection can be offered by nature to a set number of prey populations by providing refuges. The effects of prey refuges on the population dynamics are intricate and complex in nature; however, it can be considered to be constituted by two components for modeling purposes. The first component of refuge effects, which governs positive growth of prey and negatively that of predators, consists of a reduction in prey mortality owing to decreasing predation success. The second one may arise as the trade-offs and byproducts arising from the prey's hiding behavior, which could cause advantages or even be detrimental for all the 
interacting populations. González-Olivares and RamosJiliberto [8] presented prey refuges in a simple prey-predator system. Huang et al. [9] presented stability analysis of a preypredator system incorporating a prey refuge. Ma et al. [12] discussed the effect of prey refuges on a prey-predator system. Chen et al. [13] studied the prey refuge on a Leslie-Gower predator-prey model.

Extensive studies have been carried out by many researchers on refuge concept [9-30], and most of these papers consider refuge as a constant number of prey or proportional to prey. These earlier works have been taken into account that the use of refuges by a fraction of prey, or a constant number prey, is influential in existing a stabilizing effect in the dynamics of the interacting populations. This paper will test the above statement assuming that the quantity of prey in refuges is proportional to the prey. We also conduct an analysis of the dynamic properties of such a system via modification of the well-known Lotka-Volterra predatorprey model with prey self-limitation incorporating Crowley-Martin functional response [46, 47]. In this paper, we consider the refuge term proportional to prey density.

The rest of the paper is organized as follows. In Section 2, a discrete-time prey-predator model under nonoverlapping generation with refuge is formulated. Analysis of the local stability of the proposed model is dealt with in Section 3 . The bifurcation analysis of the proposed model is studied through period-doubling bifurcation and Neimark-Sacker bifurcation in Section 4. Sections 5 and 6 give Marottos chaos and control chaos procedures, respectively. The proposed model is supported with the help of numerical simulations in Section 7. Section 8 makes the conclusion to this paper.

\section{Description of the Proposed Predator- Prey Model}

The proposed model belongs to a generalized predator-prey model which incorporates logistic growth having no stage structure for neither the prey nor the predator, which is represented by the following differential equations:

$$
\begin{aligned}
& \frac{\mathrm{d} x}{\mathrm{~d} t}=r x\left(1-\frac{x}{k}\right)-c \varphi(x, y) y, \\
& \frac{\mathrm{d} y}{\mathrm{~d} t}=\mathrm{d} \varphi(x, y) y-f y,
\end{aligned}
$$

where $x$ and $y$ denote the density of prey and predator populations at any time $t$, respectively, and $r, k, c, d$, and $f$ are all positive constants and have their biological meanings accordingly. The intrinsic per capita growth rate of prey population is denoted by $r, k$ is the environmental carrying capacity of prey population, $c$ is the maximal per capita consumption rate of predators, $d$ is the efficiency with which predators convert consumed prey into new predators, and $f$ is the per capita death rate of predators. The term $\varphi(x, y)$ represents the functional response of the predator population.

According to Maynard Smith [4], there is a quantity $x_{r}$ of prey population which incorporates refuges. Thereafter, the functional response is modified to incorporate prey refuges in the above model, and the term $\varphi(x, y)$ becomes the functional response in the form of $\varphi\left(x-x_{r}, y\right)$. Based on a laboratory study of dragonfly, Crowley and Martin present functional response $\varphi(x, y)=x /(1+\alpha x)(1+\beta y)$, where $\alpha>0$ describe the handling time and $\beta>0$ is the magnitude of interference among predators. In the proposed model, we consider refuge $x_{r}=b x$, and to derive a discrete-time model let $(\mathrm{d} x / \mathrm{d} t)=\left(\left(x_{t+h}-x_{t}\right) / h\right)$ and $(\mathrm{d} y / \mathrm{d} t)=\left(\left(y_{t+h}-y_{t}\right) / h\right)$, where $x_{t}$ and $y_{t}$ are the densities of the prey and predator populations in discrete time $t$. Let $h \longrightarrow 1$ and $f=1$, then the $(n+1)$ th generation of the prey and predator populations by replacing $t$ by $n$ is obtained as follows:

$$
\begin{aligned}
& x_{n+1}=(r+1) x_{n}\left(1-\frac{r}{k(r+1)} x_{n}\right)-\frac{c(1-b) x_{n} y_{n}}{\left\{1+\alpha(1-b) x_{n}\right\}\left(1+\beta y_{n}\right)}, \\
& y_{n+1}=\frac{d(1-b) x_{n} y_{n}}{\left\{1+\alpha(1-b) x_{n}\right\}\left(1+\beta y_{n}\right)} .
\end{aligned}
$$

Let us consider $(r / k(r+1))=1$ and $(r+1)=a$, then from (2) we obtain the following proposed discrete-time predator-prey system as follows:

$$
\begin{aligned}
& x_{n+1}=a x_{n}\left(1-x_{n}\right)-\frac{c(1-b) x_{n} y_{n}}{\left\{1+\alpha(1-b) x_{n}\right\}\left(1+\beta y_{n}\right)}, \\
& y_{n+1}=\frac{d(1-b) x_{n} y_{n}}{\left\{1+\alpha(1-b) x_{n}\right\}\left(1+\beta y_{n}\right)},
\end{aligned}
$$

where $a, b, c, d, \alpha$, and $\beta$ are all positive constants. By biological meaning of the model variables, we only consider the system in the region $\Omega=\{(x, y): x \geq 0, y \geq 0\}$ in the $(x, y)$-plane.

\section{Fixed Points and Stability Analysis of the Prey-Predator System}

Fixed points of system (3) are determined by solving the following nonlinear system of equations:

$$
\begin{aligned}
& x=a x(1-x)-\frac{c(1-b) x y}{\{1+\alpha(1-b) x\}(1+\beta y)}, \\
& y=\frac{d(1-b) x y}{\{1+\alpha(1-b) x\}(1+\beta y)} .
\end{aligned}
$$

By simple calculation, we get three nonnegative fixed points as follows: (i) $P_{0}=(0,0)$, (ii) $P_{1}=((a-1 / a), 0)$, $a>1$, and (iii) $P_{2}=\left(x_{2}, y_{2}\right)$, where $x_{2}$ is positive root of the equation $A x^{3}+B x^{2}+C x+D=0$, where $A=\alpha \beta a d(1-b)$, $B=\alpha \beta d(1-a)(1-b), \quad C=\beta d(1-a)+c(1-b)(d-\alpha)$, and $D=-c$, and $y_{2}=(1 / \beta)\left[\left(d(1-b) x_{2} /\left(1+\alpha(1-b) x_{2}\right)\right)\right.$ $-1]$.

Lemma 1. Equation $A x^{3}+B x^{2}+C x+D=0$ has (i) a unique positive root if $a<1$, (ii) three positive roots if $1<a<1+(c(1-b)(d-\alpha) / \beta d), d>\alpha$, and (iii) a unique positive root if $a>1+(c(1-b)(d-\alpha) / \beta d), d>\alpha$. 
Now, the local behavior of system (3) is studied for each equilibrium point of the prey-predator system. The stability of system (3) is carried out by computing the Jacobian matrix corresponding to each equilibrium point. The Jacobian matrix $J$ for system (3) is

$$
J=\left[\begin{array}{ll}
a_{11} & a_{12} \\
a_{21} & a_{22}
\end{array}\right],
$$

where

$$
\begin{aligned}
& a_{11}=a(1-2 x)-\frac{c(1-b) y}{\{1+\alpha(1-b) x\}^{2}(1+\beta y)}=a(1-2 x)-c Q, \\
& a_{12}=-\frac{c(1-b) x}{\{1+\alpha(1-b) x\}(1+\beta y)^{2}}=-c R, \\
& a_{21}=\frac{d(1-b) y}{\{1+\alpha(1-b) x\}^{2}(1+\beta y)}=d Q, \\
& a_{22}=\frac{d(1-b) x}{\{1+\alpha(1-b) x\}(1+\beta y)^{2}}=d R,
\end{aligned}
$$

where

$$
\begin{aligned}
& Q=\frac{(1-b) y}{\{1+\alpha(1-b) x\}^{2}(1+\beta y)}, \\
& R=\frac{(1-b) x}{\{1+\alpha(1-b) x\}(1+\beta y)^{2}} .
\end{aligned}
$$

The characteristic equation of matrix $J$ is $\lambda^{2}-\operatorname{Tr}(J) \lambda+$ $\operatorname{Det}(J)=0$, where

$$
\begin{aligned}
& \operatorname{Tr}(J)=\text { Trace of matrix }=a(1-2 x)-c Q+d R \\
& \operatorname{Det}(J)=\text { Determinant of matrix }=\operatorname{ad}(1-2 x) R
\end{aligned}
$$

Hence, model (3) is a dissipative dynamical system, if $|\operatorname{ad}(1-2 x) R|<1$, is a conservative dynamical one, if and only if $|\operatorname{ad}(1-2 x) R|=1$, and is an undissipated dynamical system otherwise.

Proposition 1. The equilibrium point $P_{0}=(0,0)$ is Sink if $a<1$, Saddle if $a>1$, and Nonhyperbolic if $a=1$.

Proposition 2. The equilibrium point $P_{1}$ is Sink if $|2-a|<1$ and $|d(1-a)(1-b) /(a-\alpha(1-a)(1-b))|<1$, Source if $|2-a|>1$ and $|d(1-a)(1-b) /(a-\alpha(1-a)(1-b))|>1$, Saddle if $|2-a|>1$ and $\mid d(1-a)(1-b) /(a-\alpha(1-a)(1-$ b) $) \mid<1$ or $|2-a|<1$ and $\mid d(1-a)(1-b) /(a-\alpha(1-a)(1-$ b) $) \mid>1$, and Nonhyperbolic if $|2-a|=1$ or $\mid d(1-a)(1-$ b) $/(a-\alpha(1-a)(1-b)) \mid=1$

Proposition 3. Interior equilibrium point $P_{2}\left(x_{2}, y_{2}\right)$ is Sink if one of the following conditions holds:

(i) $x_{2}<(1 / 2)$ and $\max \left(\left((d R-c Q-1) /\left(1-2 x_{2}\right)\right)+a\right.$, $\left.\left((c Q-d R-1) /\left(1-2 x_{2}\right)\right)-a\right)<a d R<\left(1 /\left(1-2 x_{2}\right)\right)$

(ii) $x_{2}>(1 / 2)$ and $\left(1 /\left(1-2 x_{2}\right)\right)<\operatorname{adR}<\min (((d R-$ $\left.\left.c Q-1) /\left(1-2 x_{2}\right)\right)+a,\left((c Q-d R-1) /\left(1-2 x_{2}\right)\right)-a\right)$
Proposition 4. Interior equilibrium point $P_{2}\left(x_{2}, y_{2}\right)$ is Source if one of the following conditions holds:

(i) $x_{2}<(1 / 2)$ and $a d R>\max (((d R-c Q-1) /(1-$ $\left.\left.2 x_{2}\right)\right)+a,\left((c Q-d R-1) /\left(1-2 x_{2}\right)\right)-a,(1 /(1-2$ $\left.\left.x_{2}\right)\right)$

(ii) $x_{2}>(1 / 2)$ and $a d R<\min (((d R-c Q-1) /(1-$ $\left.\left.2 x_{2}\right)\right)+a,\left((c Q-d R-1) /\left(1-2 x_{2}\right)\right)-a,(1 /(1-2$ $\left.\left.x_{2}\right)\right)$ )

Proposition 5. Interior equilibrium point $P_{2}\left(x_{2}, y_{2}\right)$ is Saddle if one of the following conditions holds:

(i) $x_{2}<(1 / 2)$ and $\left((d R-c Q-1) /\left(1-2 x_{2}\right)\right)+a<$ $a d R<\left((c Q-d R-1) /\left(1-2 x_{2}\right)\right)-a$

(ii) $x_{2}>(1 / 2)$ and $\left((c Q-d R-1) /\left(1-2 x_{2}\right)\right)-a<$ $a d R<\left((d R-c Q-1) /\left(1-2 x_{2}\right)\right)+a$

Proposition 6. Interior equilibrium point $P_{2}\left(x_{2}, y_{2}\right)$ is Nonhyperbolic if one of the following conditions holds:

(i) $x_{2}<(1 / 2)$ and $\left((c Q-d R-1) /\left(1-2 x_{2}\right)\right)-a=$ $a d R \neq\left(2 /\left(1-2 x_{2}\right)\right)>\left((d R-c Q-1) /\left(1-2 x_{2}\right)\right)+a$

(ii) $x_{2}>(1 / 2)$ and $\left((c Q-d R-1) /\left(1-2 x_{2}\right)\right)-a=$ $a d R \neq\left(2 /\left(1-2 x_{2}\right)\right)<\left((d R-c Q-1) /\left(1-2 x_{2}\right)\right)+a$

(iii) $x_{2}<(1 / 2)$ and $a d R=\left(1 /\left(1-2 x_{2}\right)\right)>\max (((d R-$ $\left.c Q-1) /\left(1-2 x_{2}\right)\right)+a,\left([a(1-2 x)-c Q+d R]^{2} / 4\right.$ $\left.\left.\left(1-2 x_{2}\right)\right)\right)$

(iv) $x_{2}>(1 / 2)$ and $a d R=\left(1 /\left(1-2 x_{2}\right)\right)<\min (((d R-$ $\left.c Q-1) /\left(1-2 x_{2}\right)\right)+a,\left([a(1-2 x)-c Q+d R]^{2} / 4\right.$ $\left.\left.\left(1-2 x_{2}\right)\right)\right)$

$P D_{1}=\left\{a, b, c, d, \alpha, \beta: x_{2}<(1 / 2)\right.$ and $((c Q-d R-1) /(1-$ $\left.\left.2 x_{2}\right)\right)-a=a d R \neq\left(2 /\left(1-2 x_{2}\right)\right)>((d R-c Q-1) /(1-$ $\left.\left.\left.2 x_{2}\right)\right)+a\right\}$

$P D_{2}=\left\{a, b, c, d, \alpha, \beta: x_{2}>(1 / 2)\right.$ and $((c Q-d R-1) /$

$\left.\left(1-2 x_{2}\right)\right)-a=a d R \neq\left(2 /\left(1-2 x_{2}\right)\right)<((d R-c Q-1) /$

$\left.\left.\left(1-2 x_{2}\right)\right)+a\right\}$

$N S_{1}=\left\{a, b, c, d, \alpha, \beta: x_{2}<(1 / 2)\right.$ and $a d R=(1 /(1-$

$\left.\left.2 x_{2}\right)\right)>\max \left(\left((d R-c Q-1) /\left(1-2 x_{2}\right)\right)+a,([a(1-\right.$ $\left.\left.\left.2 x)-c Q+d R]^{2} / 4\left(1-2 x_{2}\right)\right)\right)\right\}$

$N S_{2}=\left\{a, b, c, d, \alpha, \beta: x_{2}>(1 / 2)\right.$ and $a d R=\left(1 /\left(1-2 x_{2}\right)\right)$ $<\min \left(\left((d R-c Q-1) /\left(1-2 x_{2}\right)\right)+a,([a(1-2 x)-c Q+\right.$ $\left.\left.\left.d R]^{2} / 4\left(1-2 x_{2}\right)\right)\right)\right\}$

The prey-predator model (3) undergoes period-doubling bifurcation at $P_{2}\left(x_{2}, y_{2}\right)$, when parameters vary in a small neighborhood of $P D_{1}$ or $P D_{2}$. Model (3) undergoes Neimark-Sacker bifurcation at $P_{2}\left(x_{2}, y_{2}\right)$, when parameters vary in a small neighborhood of $\mathrm{NS}_{1}$ or $\mathrm{NS}_{2}$.

\section{Bifurcation Analysis}

This section carries out an investigation of the conditions for the existence of Neimark-Sacker bifurcation (NSB) and period-doubling bifurcation (PDB) at the positive fixed point $P_{2}\left(x_{2}, y_{2}\right)$ of proposed system (3). There is an emergence of different kinds of bifurcations from the fixed 
point in dynamical systems, when a particular parameter passes through its critical value. Many dynamical properties of a system can be discussed owing to emergence of NSB and PDB. We discuss NSB and PDB for the positive fixed point $P_{2}\left(x_{2}, y_{2}\right)$ of the prey-predator system (3) taking $b$ as a bifurcation parameter.

4.1. Neimark-Sacker Bifurcation. This section presents NSB of the proposed prey-predator model (3) at $P_{2}\left(x_{2}, y_{2}\right)$ for the parameters are located in the following set:

$$
\begin{aligned}
\mathrm{NS}_{1}= & \left\{a, b, c, d, \alpha, \beta: x_{2}<\frac{1}{2} \text { and } a d R=\frac{1}{1-2 x_{2}}\right. \\
& \left.>\max \left(\frac{d R-c Q-1}{1-2 x_{2}}+a, \frac{[a(1-2 x)-c Q+d R]^{2}}{4\left(1-2 x_{2}\right)}\right)\right\} .
\end{aligned}
$$

Similar arguments can be applied to the other case:

$$
\begin{aligned}
\mathrm{NS}_{2}= & \left\{a, b, c, d, \alpha, \beta: x_{2}>\frac{1}{2} \text { and } a d R=\frac{1}{1-2 x_{2}}\right. \\
& \left.<\min \left(\frac{d R-c Q-1}{1-2 x_{2}}+a, \frac{[a(1-2 x)-c Q+d R]^{2}}{4\left(1-2 x_{2}\right)}\right)\right\} .
\end{aligned}
$$

In analyzing the NSB, $b$ is used as the bifurcation parameter. Furthermore, $b^{*}\left(\left|b^{*}\right| \lll 1\right)$ is the perturbation of $b$, and we consider a perturbation of the model as follows:

$$
\begin{aligned}
x_{n+1} & =a x_{n}\left(1-x_{n}\right)-\frac{c\left(1-\left(b+b^{*}\right)\right) x_{n} y_{n}}{\left\{1+\alpha\left(1-\left(b+b^{*}\right)\right) x_{n}\right\}\left(1+\beta y_{n}\right)} \\
& \equiv f\left(x_{n}, y_{n}, b^{*}\right), \\
y_{n+1} & =\frac{d\left(1-\left(b+b^{*}\right)\right) x_{n} y_{n}}{\left\{1+\alpha\left(1-\left(b+b^{*}\right)\right) x_{n}\right\}\left(1+\beta y_{n}\right)} \equiv g\left(x_{n}, y_{n}, b^{*}\right) .
\end{aligned}
$$

Let $u_{n}=x_{n}-x_{2}$ and $v_{n}=y_{n}-y_{2}$, then the equilibrium point $P_{2}\left(x_{2}, y_{2}\right)$ is transformed into the origin, and further expanding $f$ and $g$ as a Taylor series at $\left(u_{n}, v_{n}\right)=(0,0)$ to the third order, the proposed model (10) becomes

$$
\begin{aligned}
u_{n+1}= & \alpha_{1} u_{n}+\alpha_{2} v_{n}+\alpha_{11} u_{n}^{2}+\alpha_{12} u_{n} v_{n}+\alpha_{22} v_{n}^{2}+\alpha_{111} u_{n}^{3}+\alpha_{112} u_{n}^{2} v_{n} \\
& +\alpha_{122} u_{n} v_{n}^{2}+\alpha_{222} v_{n}^{3}+O\left(\left(\left|u_{n}\right|+\left|v_{n}\right|\right)^{4}\right), \\
v_{n+1}= & \beta_{1} u_{n}+\beta_{2} v_{n}+\beta_{11} u_{n}^{2}+\beta_{12} u_{n} v_{n}+\beta_{22} v_{n}^{2}+\beta_{111} u_{n}^{3}+\beta_{112} u_{n}^{2} v_{n} \\
& +\beta_{122} u_{n} v_{n}^{2}+\beta_{222} v_{n}^{3}+O\left(\left(\left|u_{n}\right|+\left|v_{n}\right|\right)^{4}\right),
\end{aligned}
$$

$$
\begin{aligned}
& \alpha_{1}=f_{x}\left(x_{2}, y_{2}, 0\right)=a\left(1-2 x_{2}\right)-\frac{c(1-b) y_{2}}{\left\{1+\alpha(1-b) x_{2}\right\}^{2}\left(1+\beta y_{2}\right)}, \\
& \alpha_{2}=f_{y}\left(x_{2}, y_{2}, 0\right)=-\frac{c(1-b) x_{2}}{\left\{1+\alpha(1-b) x_{2}\right\}\left(1+\beta y_{2}\right)^{2}} \text {, } \\
& \alpha_{11}=f_{x x}\left(x_{2}, y_{2}, 0\right)=-2 a+\frac{2 \alpha c(1-b)^{2} y_{2}}{\left\{1+\alpha(1-b) x_{2}\right\}^{3}\left(1+\beta y_{2}\right)} \text {, } \\
& \alpha_{12}=f_{x y}\left(x_{2}, y_{2}, 0\right)=-\frac{c(1-b)}{\left\{1+\alpha(1-b) x_{2}\right\}^{2}\left(1+\beta y_{2}\right)^{2}} \text {, } \\
& \alpha_{22}=f_{y y}\left(x_{2}, y_{2}, 0\right)=\frac{2 \beta c(1-b) x_{2}}{\left\{1+\alpha(1-b) x_{2}\right\}\left(1+\beta y_{2}\right)^{3}} \text {, } \\
& \alpha_{111}=f_{x x x}\left(x_{2}, y_{2}, 0\right)=-\frac{6 \alpha^{2} c(1-b)^{3} y_{2}}{\left\{1+\alpha(1-b) x_{2}\right\}^{4}\left(1+\beta y_{2}\right)} \text {, } \\
& \alpha_{112}=f_{x x y}\left(x_{2}, y_{2}, 0\right)=\frac{2 \alpha c(1-b)^{2}}{\left\{1+\alpha(1-b) x_{2}\right\}^{3}\left(1+\beta y_{2}\right)^{2}} \text {, } \\
& \alpha_{122}=f_{x y y}\left(x_{2}, y_{2}, 0\right)=\frac{2 \beta c(1-b)}{\left\{1+\alpha(1-b) x_{2}\right\}^{2}\left(1+\beta y_{2}\right)^{3}}, \\
& \alpha_{222}=f_{y y y}\left(x_{2}, y_{2}, 0\right)=-\frac{6 \beta^{2} c(1-b) x_{2}}{\left\{1+\alpha(1-b) x_{2}\right\}\left(1+\beta y_{2}\right)^{4}} \text {, } \\
& \beta_{1}=g_{x}\left(x_{2}, y_{2}, 0\right)=\frac{d(1-b) y_{2}}{\left\{1+\alpha(1-b) x_{2}\right\}^{2}\left(1+\beta y_{2}\right)}, \\
& \beta_{2}=g_{y}\left(x_{2}, y_{2}, 0\right)=\frac{d(1-b) x_{2}}{\left\{1+\alpha(1-b) x_{2}\right\}\left(1+\beta y_{2}\right)^{2}} \text {, } \\
& \beta_{11}=g_{x x}\left(x_{2}, y_{2}, 0\right)=-\frac{2 \alpha d(1-b)^{2} y_{2}}{\left\{1+\alpha(1-b) x_{2}\right\}^{3}\left(1+\beta y_{2}\right)}, \\
& \beta_{12}=g_{x y}\left(x_{2}, y_{2}, 0\right)=\frac{d(1-b)}{\left\{1+\alpha(1-b) x_{2}\right\}^{2}\left(1+\beta y_{2}\right)^{2}} \text {, } \\
& \beta_{22}=g_{y y}\left(x_{2}, y_{2}, 0\right)=-\frac{2 \beta d(1-b) x_{2}}{\left\{1+\alpha(1-b) x_{2}\right\}\left(1+\beta y_{2}\right)^{3}} \text {, } \\
& \beta_{111}=g_{x x x}\left(x_{2}, y_{2}, 0\right)=\frac{6 \alpha^{2} d(1-b)^{3} y_{2}}{\left\{1+\alpha(1-b) x_{2}\right\}^{4}\left(1+\beta y_{2}\right)} \text {, } \\
& \beta_{112}=g_{x x y}\left(x_{2}, y_{2}, 0\right)=-\frac{2 \alpha d(1-b)^{2}}{\left\{1+\alpha(1-b) x_{2}\right\}^{3}\left(1+\beta y_{2}\right)^{2}} \text {, } \\
& \beta_{122}=g_{x y y}\left(x_{2}, y_{2}, 0\right)=-\frac{2 \beta d(1-b)}{\left\{1+\alpha(1-b) x_{2}\right\}^{2}\left(1+\beta y_{2}\right)^{3}} \text {, } \\
& \beta_{222}=g_{y y y}\left(x_{2}, y_{2}, 0\right)=\frac{6 \beta^{2} d(1-b) x_{2}}{\left\{1+\alpha(1-b) x_{2}\right\}\left(1+\beta y_{2}\right)^{4}} \text {. }
\end{aligned}
$$

The characteristic equation associated with the linearization of model $(11)$ at $\left(u_{n}, v_{n}\right)=(0,0)$ is given by $\lambda^{2}-\operatorname{Tr}\left(J_{1}\left(b^{*}\right)\right) \lambda+\operatorname{Det}\left(J_{1}\left(b^{*}\right)\right)=0$. Roots of the characteristic equation are

where 


$$
\lambda_{1,2}\left(b^{*}\right)=\frac{\operatorname{Tr}\left(J_{1}\left(b^{*}\right)\right) \pm i \sqrt{4 \operatorname{Det}\left(J_{1}\left(b^{*}\right)\right)-\left(\operatorname{Tr}\left(J_{1}\left(b^{*}\right)\right)\right)^{2}}}{2} .
$$

From $\left|\lambda_{1,2}\left(b^{*}\right)\right|=1$, when $b^{*}=0$, we have $\left|\lambda_{1,2}\left(b^{*}\right)\right|=$ $\left[\operatorname{Det}\left(J_{1}\left(b^{*}\right)\right)\right]^{1 / 2}$ and $l=\left[\mathrm{d}\left|\lambda_{1,2}\left(b^{*}\right)\right| / \mathrm{d} b^{*}\right]_{h^{*}=0} \neq 0$.

In addition, when $b^{*}=0, \lambda_{1,2}^{i} \neq 1, i=1,2,3,4$, this is equivalent to $\operatorname{Tr}\left(J_{1}(0)\right) \neq-2,-1,1,2$.

Now to study the normal form, let $\gamma=\operatorname{Im}\left(\lambda_{1,2}\right)$ and $\delta=\operatorname{Re}\left(\lambda_{1,2}\right)$. We define $T=\left[\begin{array}{ll}0 & 1 \\ \gamma & \delta\end{array}\right]$, and using the transformation $\left[\begin{array}{l}u_{n} \\ v_{n}\end{array}\right]=T\left[\begin{array}{l}\bar{x}_{n} \\ \bar{y}_{n}\end{array}\right]$, model (11) becomes

$$
\begin{aligned}
& \bar{x}_{n+1}=\delta \bar{x}_{n}-\gamma \bar{y}_{n}+f_{1}\left(\bar{x}_{n}, \bar{y}_{n}\right), \\
& \bar{y}_{n+1}=\gamma \bar{x}_{n}+\delta \bar{y}_{n}+g_{1}\left(\bar{x}_{n}, \bar{y}_{n}\right),
\end{aligned}
$$

where the functions $f_{1}$ and $g_{1}$ denote the terms in model (14) in variables $\left(\bar{x}_{n}, \bar{y}_{n}\right)$ with the order at least two.

In order to undergo Neimark-Sacker Bifurcation, we require the following discriminatory quantity $\Omega$ to be nonzero:

$$
\Omega=-\operatorname{Re}\left[\frac{(1-2 \bar{\lambda}) \bar{\lambda}^{2}}{1-\lambda} \xi_{11} \xi_{20}\right]-\frac{1}{2}\left|\xi_{11}\right|^{2}-\left|\xi_{02}\right|^{2}+\operatorname{Re}\left(\bar{\lambda} \xi_{21}\right),
$$

where

$$
\begin{aligned}
\xi_{20}= & \frac{1}{8} \delta\left(2 \beta_{22}-\delta \alpha_{22}-\alpha_{12}+4 \gamma \alpha_{22}\right)+\frac{1}{4} \gamma \alpha_{12}+\frac{1}{8} \delta i\left(4 \gamma \alpha_{22}-2 \alpha_{22}-2 \delta \alpha_{22}\right) \\
& +\frac{1}{8} i\left(4 \gamma \beta_{22}+2 \gamma^{2} \alpha_{22}-2 \alpha_{11}\right)+\frac{1}{8} \beta_{12}+\frac{\delta \alpha_{11}-2 \beta_{11}+\delta^{3} \alpha_{22}-\delta^{2} \beta_{22}-\delta^{2} \alpha_{12}+\delta \beta_{12}}{4 \gamma}, \\
\xi_{11}= & \frac{1}{2} \gamma\left(\beta_{22}-\delta \alpha_{22}\right)+\frac{1}{2} i\left(\gamma^{2} \alpha_{22}+\alpha_{11}+\delta \alpha_{12}+\delta^{2} \alpha_{22}\right)+\frac{\beta_{11}-\delta \alpha_{11}+\delta \beta_{12}-\delta^{2} \alpha_{12}}{2 \gamma}-\frac{\delta^{2} \beta_{22}-\delta^{3} \alpha_{22}}{\gamma}, \\
\xi_{02}= & \frac{1}{4} \gamma\left(2 \delta \alpha_{22}+\alpha_{12}+\beta_{22}\right)+\frac{1}{4} i\left(\beta_{12}+2 \delta \beta_{22}-2 \delta \alpha_{12}-\alpha_{11}\right)-\frac{\beta_{11}-\delta \alpha_{11}+\delta \beta_{12}-\delta^{2} \alpha_{12}}{4 \gamma} \\
& +\frac{1}{4} \alpha_{22} i\left(\gamma^{2}-3 \delta^{2}\right)+\frac{\delta^{2} \beta_{22}-\delta^{3} \alpha_{22}}{4 \gamma}, \\
\xi_{21}= & \frac{3}{8} \beta_{222}\left(\gamma^{2}+\delta^{2}\right)+\frac{1}{8} \beta_{112}+\frac{1}{4} \delta \alpha_{112}+\frac{1}{4} \delta \beta_{122}+\alpha_{122}\left(\frac{1}{8} \gamma^{2}+\frac{3}{8} \delta^{2}-\frac{1}{4} \delta\right)+\frac{3}{8} \alpha_{111}+\frac{3}{8} \alpha_{222} i\left(\gamma^{2}+2 \delta^{2}\right)+\frac{3}{8} \alpha_{122} \gamma \delta i \\
- & \frac{1}{8} \beta_{122} \gamma i-\frac{3}{8} \beta_{222} \gamma \delta i-\frac{3 \beta_{111}-3 \delta \alpha_{111}+3 \delta \beta_{112}-3 \delta^{2} \alpha_{112}+3 \delta^{2} \beta_{122}-3 \delta^{3} \alpha_{122}+3 \delta^{3} \beta_{222}-3 \delta^{4} \alpha_{222}}{8 \gamma} i .
\end{aligned}
$$

From the above analysis we have the following result.

Theorem 1. If $\Omega \neq 0$, then model (3) undergoes NSB at $P_{2}\left(x_{2}, y_{2}\right)$ when the parameter $b^{*}$ varies in a small neighborhood of the origin. Moreover, if $\Omega<0 \quad(\Omega>0)$, then an attracting (repelling) inariant closed curve bifurcates from $P_{2}\left(x_{2}, y_{2}\right)$ for $b^{*}>0\left(b^{*}<0\right)$.
4.2. Period-Doubling Bifurcation. It is clear that one of the eigenvalues of the positive fixed point $P_{2}\left(x_{2}, y_{2}\right)$ is $\lambda_{1}=-1$ and the other $\lambda_{2}$ is neither 1 nor -1 if parameters of the model are located in the following set:

$$
\begin{aligned}
\mathrm{PD}_{1} & =\left\{a, b, c, d, \alpha, \beta: x_{2}<\frac{1}{2} \text { and } \frac{c Q-d R-1}{1-2 x_{2}}-a=a d R \neq \frac{2}{1-2 x_{2}}>\frac{d R-c Q-1}{1-2 x_{2}}+a\right\} \\
\text { or } \mathrm{PD}_{2} & =\left\{a, b, c, d, \alpha, \beta: x_{2}>\frac{1}{2} \text { and } \frac{c Q-d R-1}{1-2 x_{2}}-a=a d R \neq \frac{2}{1-2 x_{2}}<\frac{d R-c Q-1}{1-2 x_{2}}+a\right\} .
\end{aligned}
$$

Here, we discuss PDB of the proposed model (3) at $P_{2}\left(x_{2}, y_{2}\right)$, when parameters vary in a small neighborhood of $\mathrm{PD}_{1}$. Similar arguments can be applied to the other cases.
In analyzing the PDB, $b$ is used as the bifurcation parameter. Furthermore, $b^{*}\left(\left|b^{*}\right| \lll 1\right)$ is the perturbation of $b$, and we consider a perturbation of the model as follows: 


$$
\begin{aligned}
x_{n+1} & =a x_{n}\left(1-x_{n}\right)-\frac{c\left(1-\left(b+b^{*}\right)\right) x_{n} y_{n}}{\left\{1+\alpha\left(1-\left(b+b^{*}\right)\right) x_{n}\right\}\left(1+\beta y_{n}\right)} \\
& \equiv f\left(x_{n}, y_{n}, b^{*}\right), \\
y_{n+1} & =\frac{d\left(1-\left(b+b^{*}\right)\right) x_{n} y_{n}}{\left\{1+\alpha\left(1-\left(b+b^{*}\right)\right) x_{n}\right\}\left(1+\beta y_{n}\right)} \equiv g\left(x_{n}, y_{n}, b^{*}\right) .
\end{aligned}
$$

Let $u_{n}=x_{n}-x_{2}$ and $v_{n}=y_{n}-y_{2}$, then the equilibrium point $P_{2}\left(x_{2}, y_{2}\right)$ is transformed into the origin, and further expanding $f$ and $g$ as a Taylor series at $\left(u_{n}, v_{n}, b^{*}\right)=(0,0,0)$ to the third order, model (18) becomes

$$
\begin{aligned}
u_{n+1}= & \alpha_{1} u_{n}+\alpha_{2} v_{n}+\alpha_{11} u_{n}^{2}+\alpha_{12} u_{n} v_{n}+\alpha_{13} u_{n} b^{*}+\alpha_{23} v_{n} b^{*} \\
& +\alpha_{111} u_{n}^{3}+\alpha_{112} u_{n}^{2} v_{n}+\alpha_{113} u_{n}^{2} b^{*}+\alpha_{123} u_{n} v_{n} b^{*} \\
& +O\left(\left(\left|u_{n}\right|+\left|v_{n}\right|+\left|b^{*}\right|\right)^{4}\right) \\
v_{n+1}= & \beta_{1} u_{n}+\beta_{2} v_{n}+\beta_{11} u_{n}^{2}+\beta_{12} u_{n} v_{n}+\beta_{22} v_{n}^{2}+\beta_{13} u_{n} b^{*} \\
& +\beta_{23} v_{n} b^{*}+\beta_{111} u_{n}^{3} \\
& +\beta_{112} u_{n}^{2} v_{n}+\beta_{113} u_{n}^{2} b^{*}+\beta_{123} u_{n} v_{n} b^{*}+\beta_{223} v_{n}^{2} b^{*} \\
& +O\left(\left(\left|u_{n}\right|+\left|v_{n}\right|+\left|b^{*}\right|\right)^{4}\right),
\end{aligned}
$$

where $\alpha_{1}=f_{x}\left(x_{2}, y_{2}, 0\right), \alpha_{2}=f_{y}\left(x_{2}, y_{2}, 0\right), \alpha_{11}=f_{x x}\left(x_{2}\right.$, $\left.y_{2}, 0\right), \alpha_{12}=f_{x y}\left(x_{2}, y_{2}, 0\right), \alpha_{13}=f_{x b^{*}}\left(x_{2}, y_{2}, 0\right), \alpha_{23}=f_{y b^{*}}$ $\left(x_{2}, y_{2}, 0\right), \quad \alpha_{111}=f_{x x x}\left(x_{2}, y_{2}, 0\right), \quad \alpha_{112}=f_{x x y}\left(x_{2}, y_{2}, 0\right)$, $\alpha_{113}=f_{x x b^{*}}\left(x_{2}, y_{2}, 0\right), \alpha_{123}=f_{x y b^{*}}\left(x_{2}, y_{2}, 0\right), \beta_{1}=g_{x}\left(x_{2}\right.$, $\left.y_{2}, 0\right), \beta_{2}=g_{y}\left(x_{2}, y_{2}, 0\right), \beta_{11}=g_{x x}\left(x_{2}, y_{2}, 0\right), \beta_{12}=g_{x y}\left(x_{2}\right.$, $\left.y_{2}, 0\right), \beta_{22}=g_{y y}\left(x_{2}, y_{2}, 0\right), \beta_{13}=g_{x b^{*}}\left(x_{2}, y_{2}, 0\right), \beta_{23}=g_{y b^{*}}$ $\left(x_{2}, y_{2}, 0\right), \quad \beta_{111}=g_{x x x}\left(x_{2}, y_{2}, 0\right), \quad \beta_{113}=g_{x x b^{*}}\left(x_{2}, y_{2}, 0\right)$, $\beta_{123}=g_{x y b^{*}}\left(x_{2}, y_{2}, 0\right)$, and $\beta_{223}=g_{y y b^{*}}\left(x_{2}, y_{2}, 0\right)$.

We define $T=\left[\begin{array}{cc}\alpha_{2} & \alpha_{2} \\ -1-\alpha_{1} & \lambda_{2}-\alpha_{1}\end{array}\right]$, and it is obvious that $T$ is invertible. Using the transformation $\left[\begin{array}{l}u_{n} \\ v_{n}\end{array}\right]=T\left[\begin{array}{l}\bar{x}_{n} \\ \bar{y}_{n}\end{array}\right]$, then model (19) becomes

$$
\begin{aligned}
& x_{n+1}=-\bar{x}_{n}+f_{1}\left(u_{n}, v_{n}, b^{*}\right), \\
& \bar{y}_{n+1}=\lambda_{2} \bar{y}_{n}+g_{1}\left(u_{n}, v_{n}, b^{*}\right),
\end{aligned}
$$

where the functions $f_{1}$ and $g_{1}$ denote the terms in model (20) in variables $\left(u_{n}, v_{n}, b^{*}\right)$ with the order at least two.

From the center manifold theorem, we know that there exists a center manifold $W^{c}(0,0,0)$ of model $(20)$ at $(0,0)$ in a small neighborhood of $b^{*}=0$, which can be approximately described as follows:

$$
\begin{aligned}
W^{c}(0,0,0)= & \left\{\left(\bar{x}_{n}, \bar{y}_{n}, b^{*}\right) \varepsilon R^{3}: \bar{y}_{n+1}=\bar{\alpha}_{1} \bar{x}_{n}^{2}+\bar{\alpha}_{2} \bar{x}_{n} b^{*}\right. \\
& \left.+O\left(\left(\left|\bar{x}_{n}\right|+\left|b^{*}\right|\right)^{3}\right)\right\},
\end{aligned}
$$

where

$$
\begin{aligned}
\bar{\alpha}_{1}= & \frac{\alpha_{2}\left[\left(1+\alpha_{1}\right) \alpha_{11}+\alpha_{2} \beta_{11}\right]}{1-\lambda_{2}^{2}}+\frac{\beta_{22}\left(1+\alpha_{1}\right)^{2}}{1-\lambda_{2}^{2}} \\
& -\frac{\left(1+\alpha_{1}\right)\left[\alpha_{12}\left(1+\alpha_{1}\right)+\alpha_{2} \beta_{12}\right]}{1-\lambda_{2}^{2}}, \\
\bar{\alpha}_{2}= & \frac{\left(1+\alpha_{1}\right)\left[\alpha_{23}\left(1+\alpha_{1}\right)+\alpha_{2} \beta_{23}\right]}{\alpha_{2}\left(1+\lambda_{2}\right)^{2}}-\frac{\left(1+\alpha_{1}\right)\left[\alpha_{13}+\alpha_{2} \beta_{13}\right]}{\left(1+\lambda_{2}\right)^{2}} .
\end{aligned}
$$

Model (20) which is restricted to center manifold $W^{c}(0,0,0)$ has the following form:

$$
\begin{aligned}
\bar{x}_{n+1}= & -\bar{x}_{n}+h_{1} \bar{x}_{n}^{2}+h_{2} \bar{x}_{n} b^{*}+h_{3} \bar{x}_{n}^{2} b^{*}+h_{4} \bar{x}_{n} b^{* 2}+h_{5} \bar{x}_{n}^{3} \\
& +O\left(\left(\left|\bar{x}_{n}\right|+\left|b^{*}\right|\right)^{3}\right) \equiv F\left(\bar{x}_{n}, b^{*}\right),
\end{aligned}
$$

where

$$
\begin{aligned}
h_{1}= & \frac{\bar{\alpha}_{2}\left[\left(\lambda_{2}-\bar{\alpha}_{1}\right) \alpha_{11}-\bar{\alpha}_{2} \beta_{11}\right]}{1+\lambda_{2}}-\frac{\beta_{22}\left(1+\bar{\alpha}_{1}\right)^{2}}{1+\lambda_{2}}-\frac{\left(1+\bar{\alpha}_{1}\right)\left[\left(\lambda_{2}-\bar{\alpha}_{1}\right) \alpha_{12}-\bar{\alpha}_{2} \beta_{12}\right]}{1+\lambda_{2}}, \\
h_{2}= & \frac{\left(\lambda_{2}-\bar{\alpha}_{1}\right) \alpha_{13}-\bar{\alpha}_{2} \beta_{13}}{1+\lambda_{2}}-\frac{\left(1+\bar{\alpha}_{1}\right)\left[\left(\lambda_{2}-\bar{\alpha}_{1}\right) \alpha_{23}-\bar{\alpha}_{2} \beta_{23}\right]}{\bar{\alpha}_{2}\left(1+\lambda_{2}\right)}, \\
h_{3}= & \frac{\left[\left(\lambda_{2}-\alpha_{1}\right) \alpha_{23}-\alpha_{2} \beta_{23}\right]\left(\lambda_{2}-\alpha_{1}\right) \bar{\alpha}_{1}}{\alpha_{2}\left(1+\lambda_{2}\right)}+\frac{\left(\lambda_{2}-\alpha_{1}\right) \bar{\alpha}_{1} \alpha_{13}-\alpha_{2} \beta_{13}+\alpha_{2}\left[\left(\lambda_{2}-\alpha_{1}\right) \alpha_{113}-\alpha_{2} \beta_{113}\right]-\beta_{223}\left(1+\alpha_{1}\right)^{2}}{1+\lambda_{2}} \\
& +\frac{2 \alpha_{2} \bar{\alpha}_{2}\left[\left(\lambda_{2}-\alpha_{1}\right) \alpha_{11}-\alpha_{2} \beta_{11}\right]}{1+\lambda_{2}}-\frac{\left(1+\alpha_{1}\right)\left[\left(\lambda_{2}-\alpha_{1}\right) \alpha_{123}-\alpha_{2} \beta_{123}\right]}{1+\lambda_{2}}-\frac{2 \beta_{22} \bar{\alpha}_{2}\left(1+\alpha_{1}\right)\left(\lambda_{2}-\alpha_{1}\right)}{1+\lambda_{2}} \\
& +\frac{\bar{\alpha}_{2}\left[\left(\lambda_{2}-\alpha_{1}\right) \alpha_{12}-\alpha_{2} \beta_{12}\right]\left(\lambda_{2}-1-2 \alpha_{1}\right)}{1+\lambda_{2}},
\end{aligned}
$$




$$
\begin{aligned}
h_{4}= & \frac{\bar{\alpha}_{2}\left[\left(\lambda_{2}-\alpha_{1}\right) \alpha_{13}-\alpha_{2} \beta_{13}\right]}{1+\lambda_{2}}+\frac{\left[\left(\lambda_{2}-\alpha_{1}\right) \alpha_{23}-\alpha_{2} \beta_{23}\right]\left(\lambda_{2}-\alpha_{1}\right) \bar{\alpha}_{2}}{\alpha_{2}\left(1+\lambda_{2}\right)}+\frac{2 \alpha_{2} \bar{\alpha}_{2}\left[\left(\lambda_{2}-\alpha_{1}\right) \alpha_{11}-\alpha_{2} \beta_{11}\right]}{1+\lambda_{2}} \\
& +\frac{2 \beta_{22} \bar{\alpha}_{2}\left(1+\alpha_{1}\right)\left(\lambda_{2}-\alpha_{1}\right)}{1+\lambda_{2}}+\frac{\bar{\alpha}_{2}\left[\left(\lambda_{2}-\alpha_{1}\right) \alpha_{12}-\alpha_{2} \beta_{12}\right]\left(\lambda_{2}-1-2 \alpha_{1}\right)}{1+\lambda_{2}}, \\
h_{5}= & \frac{2 \alpha_{2} \bar{\alpha}_{1}\left[\left(\lambda_{2}-\alpha_{1}\right) \alpha_{11}-\alpha_{2} \beta_{11}\right]+\bar{\alpha}_{2}^{2}\left[\left(\lambda_{2}-\alpha_{1}\right) \alpha_{111}-\alpha_{2} \beta_{111}\right]}{1+\lambda_{2}}+\frac{2 \beta_{22} \bar{\alpha}_{1}\left(\lambda_{2}-\alpha_{1}\right)\left(1+\alpha_{1}\right)}{1+\lambda_{2}} \\
& +\frac{\left[\left(\lambda_{2}-\alpha_{1}\right) \alpha_{11}-\alpha_{2} \beta_{11}\right]\left(\lambda_{2}-1-2 \alpha_{1}\right) \bar{\alpha}_{1}}{1+\lambda_{2}}-\frac{\bar{\alpha}_{2}\left(1+\alpha_{1}\right)\left[\left(\lambda_{2}-\alpha_{1}\right) \alpha_{112}-\alpha_{2} \beta_{112}\right]}{1+\lambda_{2}} .
\end{aligned}
$$

For flip bifurcation, we require the two discriminatory quantities $\xi_{1}$ and $\xi_{2}$ to be nonzero:

$$
\begin{aligned}
& \xi_{1}=\left.\left(\frac{\partial^{2} F}{\partial \bar{x} \partial b^{*}}+\frac{1}{2} \frac{\partial F}{\partial b^{*}} \frac{\partial^{2} F}{\partial \bar{x}^{2}}\right)\right|_{(0,0)}, \\
& \xi_{2}=\left.\left(\frac{1}{6} \frac{\partial^{3} F}{\partial \bar{x}^{3}}+\left(\frac{1}{2} \frac{\partial^{2} F}{\partial \bar{x}^{2}}\right)^{2}\right)\right|_{(0,0)} .
\end{aligned}
$$

Finally, from the above analysis, we have the following result.

Theorem 2. If $\xi_{1} \neq 0$ and $\xi_{2} \neq 0$, then model (3) undergoes $P D B$ at $P_{2}\left(x_{2}, y_{2}\right)$ when the parameter $b$ varies. Moreover, if $\xi_{2}>0\left(\xi_{2}<0\right)$, then the period-2 points that bifurcation from $P_{2}\left(x_{2}, y_{2}\right)$ are stable (unstable).

\section{Existence of Marottos Chaos}

This section presents chaotic nature of system (3) in the sense of Marrotto.

Definition 1. Let the function $F: R^{n} \longrightarrow R^{n}$ be differentiable in $B_{r}(Z)$. The point $Z \in R^{n}$ is an expanding fixed point of $F$ in $B_{r}(Z)$, if $F(Z)=Z$ and all eigenvalues of $D F(X)$ exceed 1 in norm for all $X \in B_{r}(Z)$.

Definition 2. Assume that $Z$ is an expanding fixed point of $F$ in $B_{r}(Z)$ for some $r>0$. Then, $Z$ is said to be a snap-back repeller of $F$ if there exists a point $X_{0} \in B_{r}(Z)$ with $X_{0} \neq Z$, $F^{M}\left(X_{0}\right)=Z$, and $D F^{M}(X 0) \neq 0$ for some positive integer $M$.

Theorem 3. If $P_{2} \in U_{2}=\left\{(x, y): 4 a d\left(1-2 x_{2}\right) R_{2}>\max \right.$ $\left.\left(4,\left[a\left(1-2 x_{2}\right)-c Q_{2}+d R_{2}\right]^{2}\right)\right\}$, then $P_{2}$ is an expanding fixed point of $F$.

Proof. For map,

$$
\begin{aligned}
F\left(X_{n}\right) & =\left(\begin{array}{c}
a x_{n}\left(1-x_{n}\right)-\frac{c(1-b) x_{n} y_{n}}{\left\{1+\alpha(1-b) x_{n}\right\}\left(1+\beta y_{n}\right)} \\
\frac{d(1-b) x_{n} y_{n}}{\left\{1+\alpha(1-b) x_{n}\right\}\left(1+\beta y_{n}\right)}
\end{array}\right), \\
X_{n} & =\left(x_{n} y_{n}\right)^{T} .
\end{aligned}
$$

The eigenvalues corresponding with the fixed point $P_{2}=$ $\left(x_{2}, y_{2}\right)$ are given by

$$
\lambda_{1,2}=\frac{-p\left(x_{2}, y_{2}\right) \pm \sqrt{p^{2}\left(x_{2}, y_{2}\right)-4 q\left(x_{2}, y_{2}\right)}}{2},
$$

where

$$
\begin{aligned}
p\left(x_{2}, y_{2}\right) & =-\left[a\left(1-2 x_{2}\right)-c Q_{2}+d R_{2}\right], \\
q\left(x_{2}, y_{2}\right) & =a d\left(1-2 x_{2}\right) R_{2}, \\
R_{2} & =\frac{(1-b) x_{2}}{\left\{1+\alpha(1-b) x_{2}\right\}\left(1+\beta y_{2}\right)^{2}}, \\
Q_{2} & =\frac{(1-b) y_{2}}{\left\{1+\alpha(1-b) x_{2}\right\}^{2}\left(1+\beta y_{2}\right)} .
\end{aligned}
$$

Therefore, the fixed point $P_{2}\left(x_{2}, y_{2}\right)$ has a pair of complex eigenvalues, and the norm of them exceeds unity if

$$
\begin{aligned}
& p^{2}\left(x_{2}, y_{2}\right)-4 q\left(x_{2}, y_{2}\right)<0 \text { and } q\left(x_{2}, y_{2}\right)-1>0 \\
& \text { i.e., } 4 a d\left(1-2 x_{2}\right) R_{2}>\max \left(4,\left[a\left(1-2 x_{2}\right)-c Q_{2}+d\right.\right. \\
& \left.\left.R_{2}\right]^{2}\right) .
\end{aligned}
$$

Thus, we can state the following theorem.

Theorem 4. $P_{2}\left(x_{2}, y_{2}\right)$ is a snap-back repeller in $U_{2}$.

Proof. According to the definition of a snap-back repeller, one needs to find one point $P_{*}\left(x_{0}, y_{0}\right) \in U_{2}$ such that $P_{*} \neq P_{2}, F^{M}\left(P_{*}\right)=P_{2}$, and $\left|D F^{M}\left(P_{*}\right)\right| \neq 0$, for some positive integer $M$, where Map $F$ is defined by (3).

To proceed, notice that 


$$
\begin{aligned}
& x_{1}=a x_{0}\left(1-x_{0}\right)-\frac{c(1-b) x_{0} y_{0}}{\left\{1+\alpha(1-b) x_{0}\right\}\left(1+\beta y_{0}\right)}, \\
& y_{1}=\frac{d(1-b) x_{0} y_{0}}{\left\{1+\alpha(1-b) x_{0}\right\}\left(1+\beta y_{0}\right)}, \\
& x_{2}=a x_{1}\left(1-x_{1}\right)-\frac{c(1-b) x_{1} y_{1}}{\left\{1+\alpha(1-b) x_{1}\right\}\left(1+\beta y_{1}\right)}, \\
& y_{2}=\frac{d(1-b) x_{1} y_{1}}{\left\{1+\alpha(1-b) x_{1}\right\}\left(1+\beta y_{1}\right)} .
\end{aligned}
$$

Now, a map $F^{2}$ has been constructed to map the point $P_{*}=\left(x_{0}, y_{0}\right)$ to the fixed point $P_{2}\left(x_{2}, y_{2}\right)$ after two iterations if there are solutions different from $P_{2}$ for equations (29) and (30). The solutions different from $P_{2}$ for equation (30) satisfy the following equation:

$$
\begin{aligned}
& x_{1}=\frac{y_{2}\left(1+\beta y_{1}\right)}{(1-b)\left[d y_{1}-\alpha y_{2}\left(1+\beta y_{1}\right)\right]}, \\
& y_{1}=\frac{\left[1+\alpha(1-b) x_{1}\right]\left(1+\beta y_{1}\right)\left[a x_{1}\left(1-x_{1}\right)-x_{2}\right]}{c(1-b) x_{1}} .
\end{aligned}
$$

Substituting $x_{1}$ and $y_{1}$ into equation (29) and solving $x_{0}, y_{0}$, we have

$$
\begin{aligned}
& x_{0}=\frac{y_{1}\left(1+\beta y_{0}\right)}{(1-b)\left[d y_{0}-\alpha y_{1}\left(1+\beta y_{0}\right)\right]}, \\
& y_{0}=\frac{\left[1+\alpha(1-b) x_{0}\right]\left(1+\beta y_{0}\right)\left[a x_{0}\left(1-x_{0}\right)-x_{1}\right]}{c(1-b) x_{0}} .
\end{aligned}
$$

By simple calculations, we obtain

$$
\begin{aligned}
\left|D F^{2}\left(P_{*}\right)\right|= & {\left[a(B-2 A B)-\frac{[1+\alpha(1-b) A][1+\beta D][B D+A E] c(1-b)-c(1-b) A D[[\alpha(1-b) B][1+\beta D]+[1+\alpha(1-b) A] \beta E]}{[1+\alpha(1-b) A]^{2}[1+\beta D]^{2}}\right] } \\
& \times\left[\frac{[1+\alpha(1-b) A][1+\beta D] d(1-b)[C D+A F]-d(1-b) A D[[\alpha(1-b) C][1+\beta D]+[1+\alpha(1-b) A] \beta F]}{[1+\alpha(1-b) A]^{2}[1+\beta D]^{2}}\right] \\
& -\left[a(C-2 A C)-\frac{[1+\alpha(1-b) A][1+\beta D] c(1-b)[C D+A F]-c(1-b) A D[[\alpha(1-b) C][1+\beta D]+[1+\alpha(1-b) A] \beta F]}{[1+\alpha(1-b) A]^{2}[1+\beta D]^{2}}\right] \\
& \times\left[\frac{[1+\alpha(1-b) A][1+\beta D][B D+A E] d(1-b)-d(1-b) A D[[\alpha(1-b) B][1+\beta D]+[1+\alpha(1-b) A] \beta E]}{[1+\alpha(1-b) A]^{2}[1+\beta D]^{2}}\right],
\end{aligned}
$$

where

$$
\begin{aligned}
& A=a x(1-x)-\frac{c(1-b) x y}{\{1+\alpha(1-b) x\}(1+\beta y)}, \\
& B=a(1-2 x)-\frac{c y}{\{1+\alpha(1-b) x\}^{2}(1+\beta y)}, \\
& C=-\frac{c(1-b) x}{\{1+\alpha(1-b) x\}(1+\beta y)^{2}}, \\
& D=\frac{d(1-b) x y}{\{1+\alpha(1-b) x\}(1+\beta y)}, \\
& E=\frac{d y}{\{1+\alpha(1-b) x\}^{2}(1+\beta y)}, \\
& F=\frac{d(1-b) x}{\{1+\alpha(1-b) x\}(1+\beta y)^{2}} .
\end{aligned}
$$

The solutions of equations (31) and (32) will farther subject to $\left(x_{0}, y_{0}\right),\left(x_{1}, y_{1}\right) \neq\left(x_{2}, y_{2}\right),\left(x_{0}, y_{0}\right) \in U_{2}$ and $\left|D F^{2}\left(P_{*}\right)\right| \neq 0$, then $P_{2}\left(x_{2}, y_{2}\right)$ is a snap-back repeller in $U_{2}$.

Thus, the following theorem is established.
Theorem 5. If $4 a d\left(1-2 x_{2}\right) R_{2}>\max \left(4,\left[a\left(1-2 x_{2}\right)-\right.\right.$ $\left.\left.c Q_{2}+d R_{2}\right]^{2}\right)$, the solutions $\left(x_{1}, y_{1}\right)$ and $\left(x_{0}, y_{0}\right)$ of equations (31) and (32) satisfy in addition $\left(x_{0}, y_{0}\right),\left(x_{1}, y_{1}\right) \neq\left(x_{2}, y_{2}\right)$, $\left(x_{0}, y_{0}\right) \in U_{2},\left(x_{0}, y_{0}\right)=(0,0)$, and $\left|D F^{2}\left(P_{*}\right)\right| \neq 0$, then $P_{2}\left(x_{2}, y_{2}\right)$ is a snap-back repeller of Map (3), and hence Map (3) is chaotic in the sense of Marotto.

\section{Chaos Control of the Proposed System}

Several methods can be used for obtaining chaos control in discrete-time models. A few of these methods are the state feedback method, pole-placement technique, and hybrid control method. We give a feedback control method to stabilize chaotic orbits at an unstable positive fixed point of the prey-predator system (3), with the following controlled form:

$$
\begin{aligned}
& x_{n+1}=a x_{n}\left(1-x_{n}\right)-\frac{c(1-b) x_{n} y_{n}}{\left\{1+\alpha(1-b) x_{n}\right\}\left(1+\beta y_{n}\right)}+S, \\
& y_{n+1}=\frac{d(1-b) x_{n} y_{n}}{\left\{1+\alpha(1-b) x_{n}\right\}\left(1+\beta y_{n}\right)}
\end{aligned}
$$




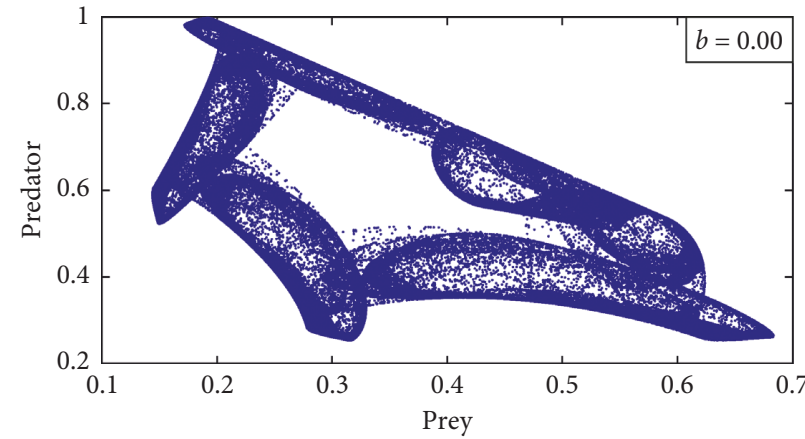

(a)

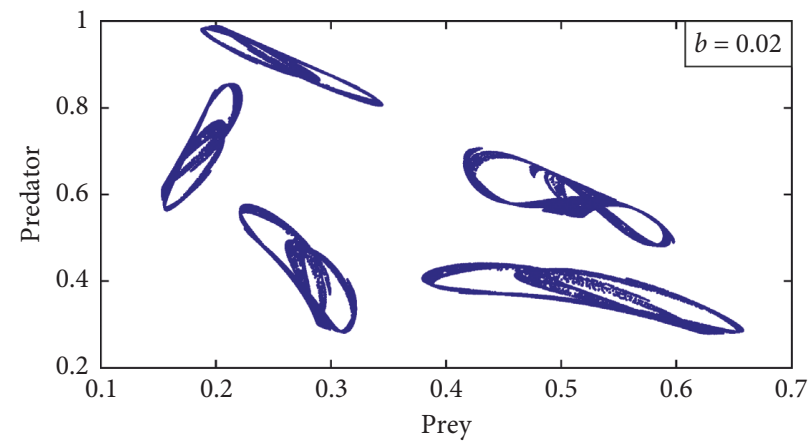

(c)

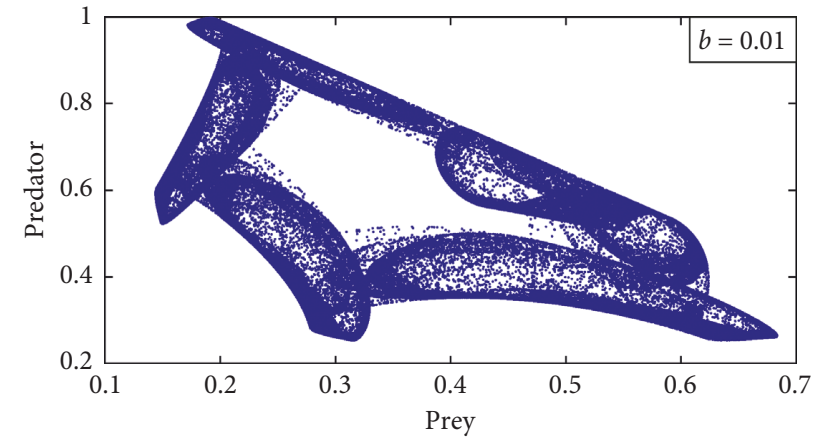

(b)

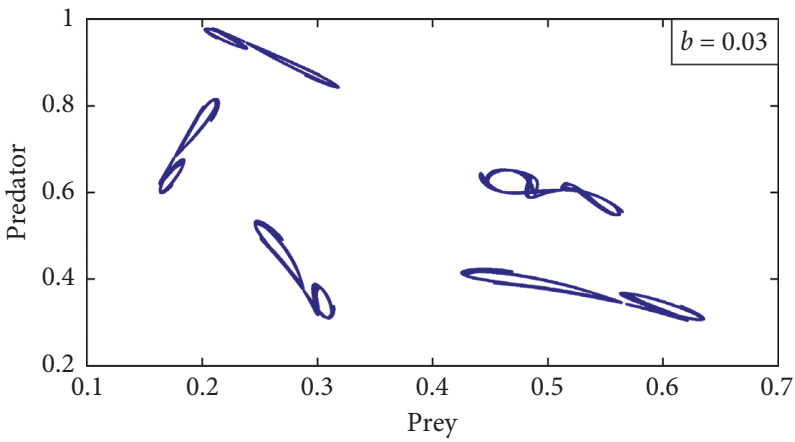

(d)

Figure 1: Phase portraits for $b=0.0,0.01,0.02,0.03$.

with the following feedback control law as the control force:

$$
S=-q_{1}\left(x_{n}-x_{2}\right)-q_{2}\left(y_{n}-y_{2}\right),
$$

where $q_{1}$ and $q_{2}$ are the feedback gain and $\left(x_{2}, y_{2}\right)$ is a positive fixed point of the model.

The Jacobian matrix $J$ for system $(35)$ at $\left(x_{2}, y_{2}\right)$ is

$$
J=\left[\begin{array}{cc}
a_{11}-q_{1} & a_{12}-q_{2} \\
a_{21} & a_{22}
\end{array}\right],
$$

where

$$
\begin{aligned}
& a_{11}=a(1-2 x)-\frac{c(1-b) y}{\{1+\alpha(1-b) x\}^{2}(1+\beta y)}, \\
& a_{12}=-\frac{c(1-b) x}{\{1+\alpha(1-b) x\}(1+\beta y)^{2}}, \\
& a_{21}=\frac{d(1-b) y}{\{1+\alpha(1-b) x\}^{2}(1+\beta y)}, \\
& a_{22}=\frac{d(1-b) x}{\{1+\alpha(1-b) x\}(1+\beta y)^{2}} .
\end{aligned}
$$

The corresponding characteristic equation of matrix $J$ is

$$
\lambda^{2}-\left(a_{11}+a_{22}-q_{1}\right) \lambda+a_{22}\left(a_{11}-q_{1}\right)-a_{21}\left(a_{12}-q_{2}\right) \text {. }
$$

Let $\lambda_{1}$ and $\lambda_{2}$ are the eigenvalues of the above characteristic equation:

$$
\begin{aligned}
\lambda_{1}+\lambda_{2} & =a_{11}+a_{22}-q_{1}, \\
\lambda_{1} \lambda_{2} & =a_{22}\left(a_{11}-q_{1}\right)-a_{21}\left(a_{12}-q_{2}\right) .
\end{aligned}
$$

The lines of marginal stability are determined by solving the equations $\lambda_{1}= \pm 1$ and $\lambda_{1} \lambda_{2}=1$. These conditions guarantee that the eigenvalues $\lambda_{1}$ and $\lambda_{2}$ have modulus less than 1.

Suppose $\lambda_{1} \lambda_{2}=1$, from (40), we have line $l_{1}$ as follows:

$$
a_{22} q_{1}-a_{21} q_{2}=a_{22} a_{11}-a_{21} a_{12}-1 \text {. }
$$

Suppose $\lambda_{1}= \pm 1$, from (40), we have line $l_{2}$ and $l_{3}$ as follows:

$$
\begin{aligned}
& \left(1-a_{22}\right) q_{1}+a_{21} q_{2}=a_{11}+a_{22}-1-a_{22} a_{11}+a_{21} a_{12}, \\
& \left(1+a_{22}\right) q_{1}-a_{21} q_{2}=a_{11}+a_{22}+1+a_{22} a_{11}-a_{21} a_{12} .
\end{aligned}
$$

The stable eigenvalues lie within a triangular region by line $l_{1}, l_{2}$, and $l_{3}$.

\section{Numerical Simulations}

This section presents the qualitative dynamical behaviors of the system by presenting Lyapunov exponent, bifurcation diagram, and phase plane for specific parameter values. We present the numerical simulation to observe rich dynamics of our model, so we consider the hypothetical parameters values of the proposed discrete prey-predator system. The value of the parameters are consider as $a=4.2, b=0.12$, $c=3.0, d=3.5, \alpha=0.1$, and $\beta=0.1$ with initial population 


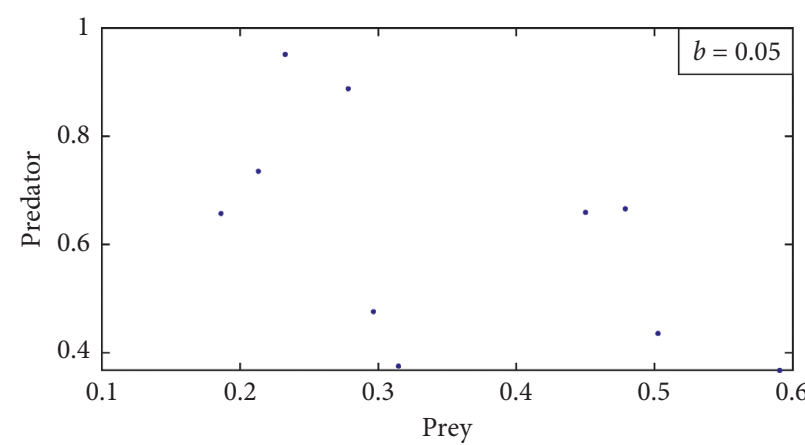

(a)

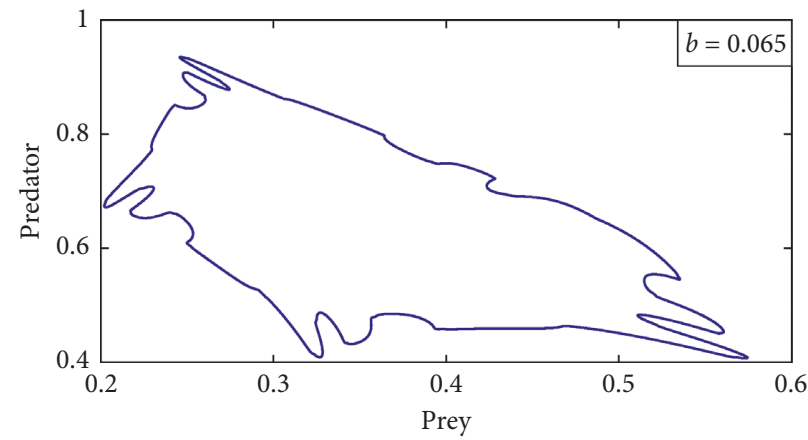

(c)

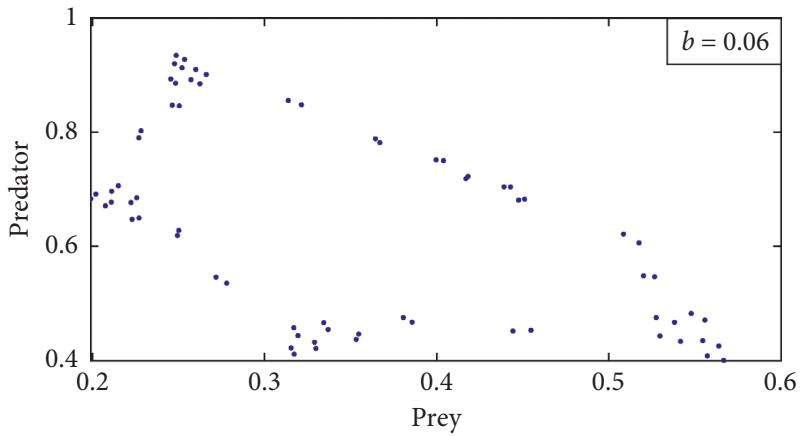

(b)

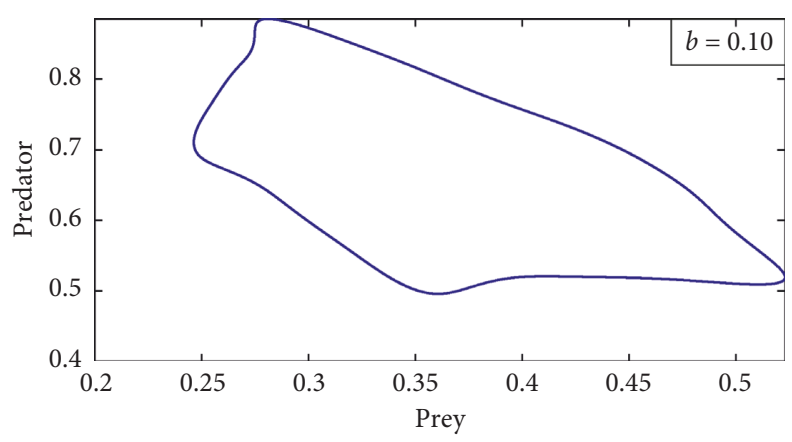

(d)

FIGURE 2: Phase portraits for $b=0.05,0.06,0.065,0.10$.

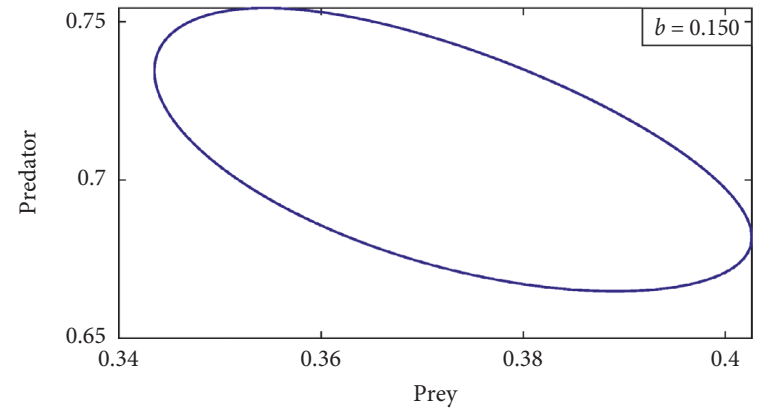

(a)

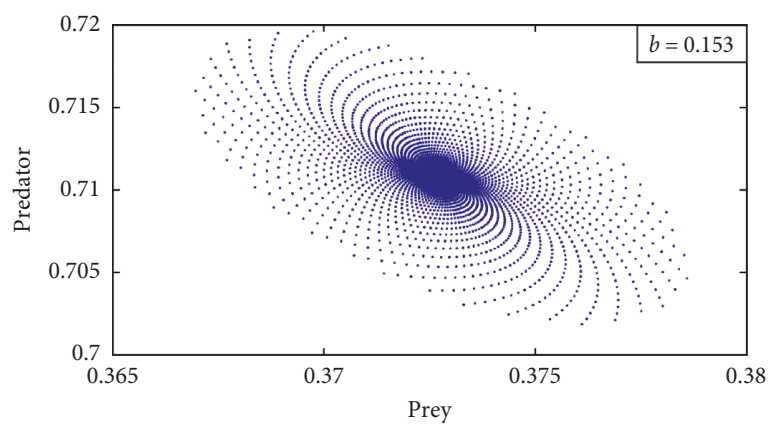

(c)

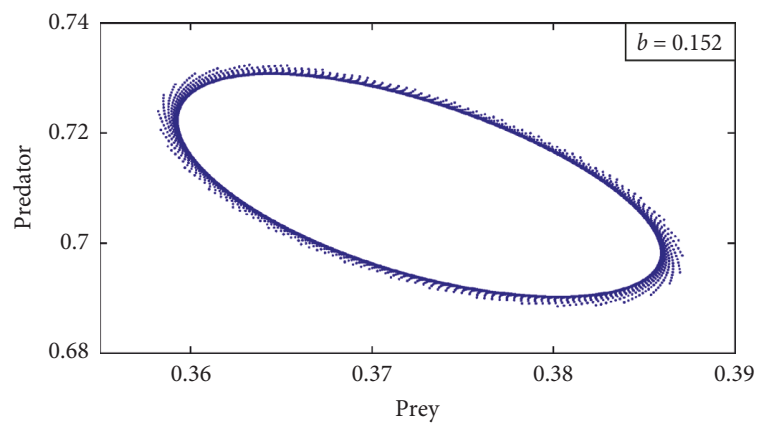

(b)

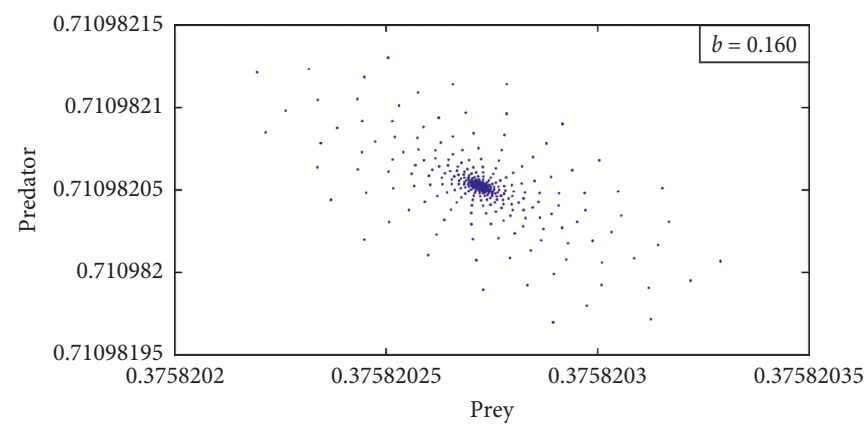

(d)

Figure 3: Phase portraits for $b=0.150,0.152,0.153,0.160$. 


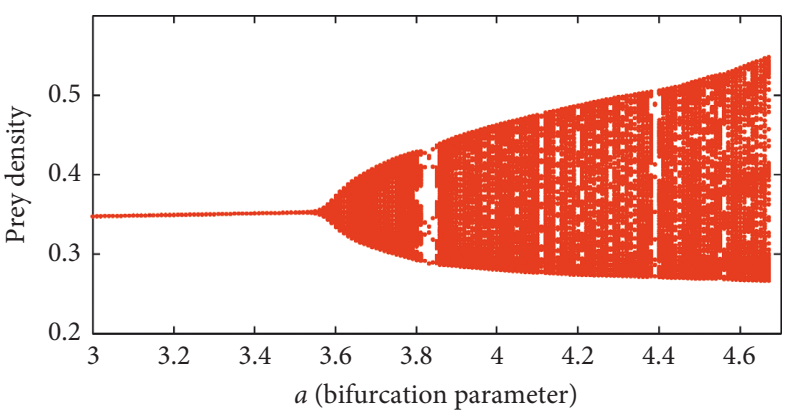

(a)

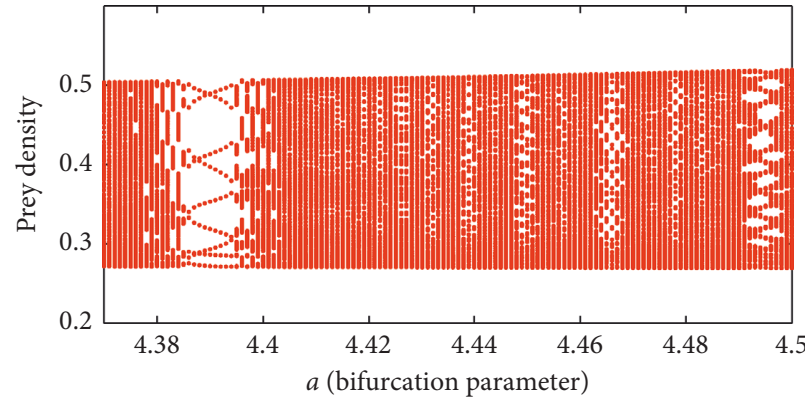

(c)

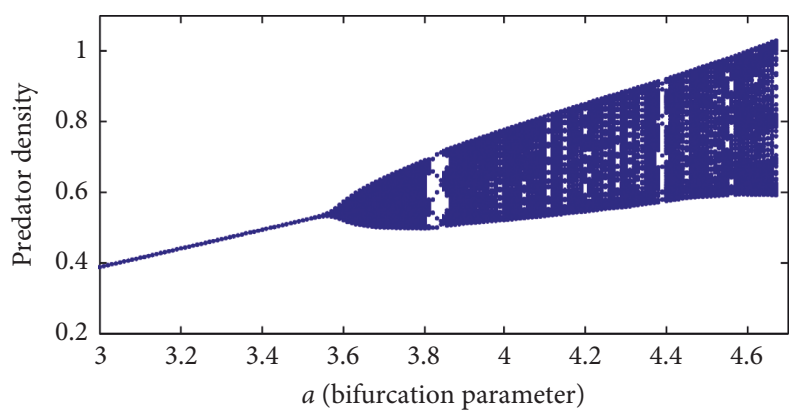

(b)

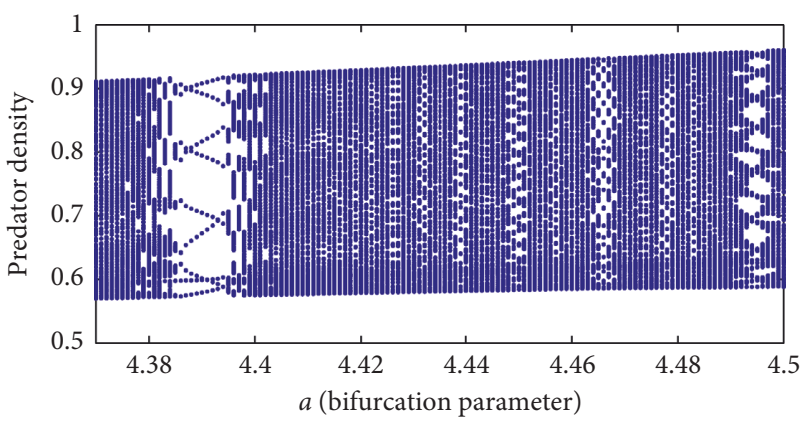

(d)

Figure 4: Orbit diagram of species for parameter $a$ and local amplification.

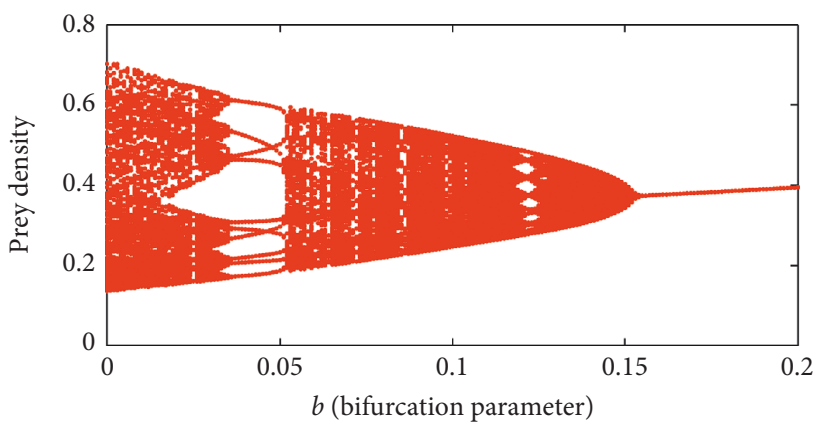

(a)

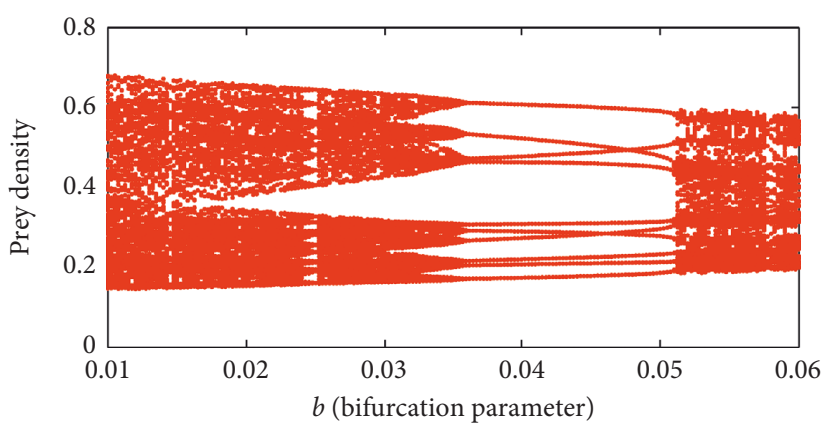

(c)

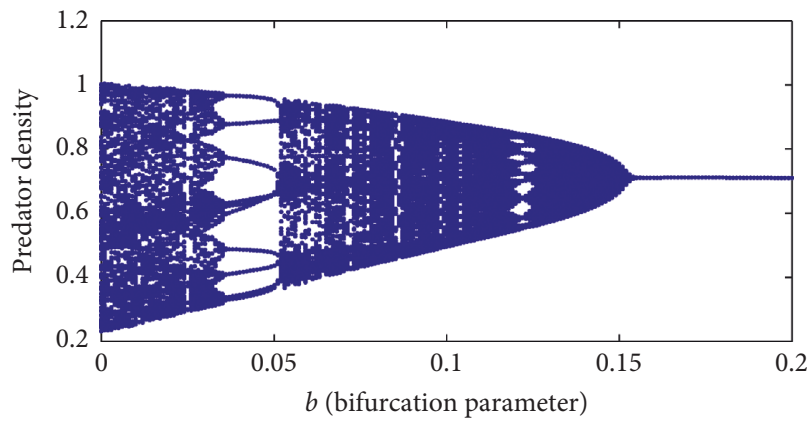

(b)

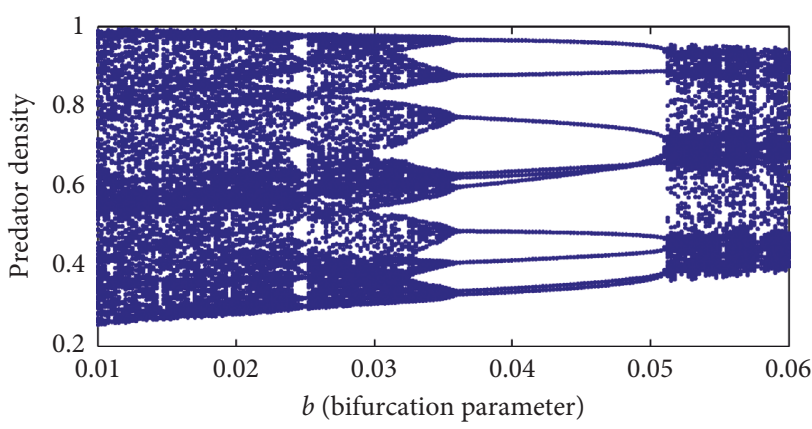

(d)

FIgURe 5: Orbit diagram of species for parameter $b$ and local amplification.

$(0.6,0.3)$ for simulation and graphical presentation of different dynamical observations. Figures 1-3 are iterative plots for different refuge parameters, and we observed that refuge has a stabilizing effect. Figures $4-9$ are bifurcation diagrams and corresponding local amplification diagram of the prey and predator for varying parameters $a, b, c, d, \alpha$, and $\beta$, respectively.

The phase portraits associated with Figure 5 are displayed in Figures 1-3. A chaotic attractor for $b=0.00$ and 0.01 . A five-pieces chaotic attractor for $b=0.02$ and 0.03 . 


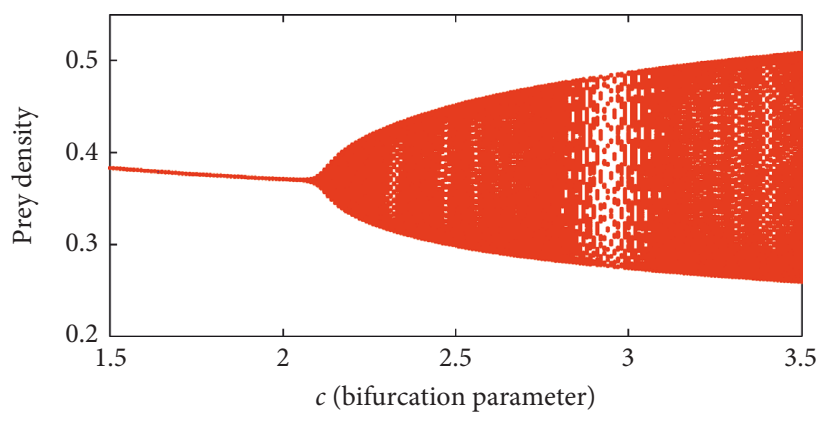

(a)

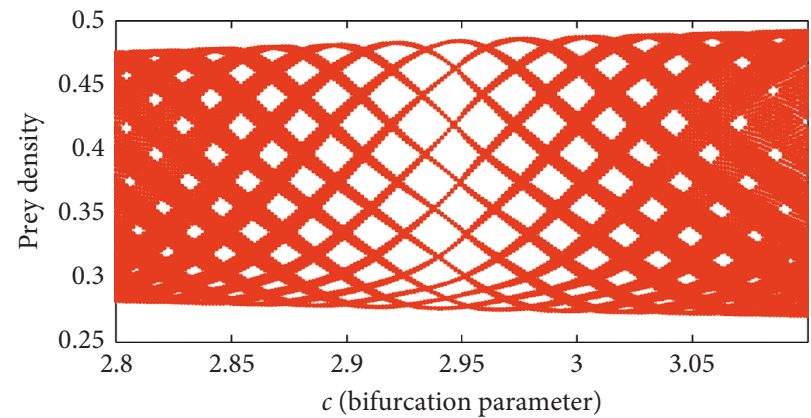

(c)

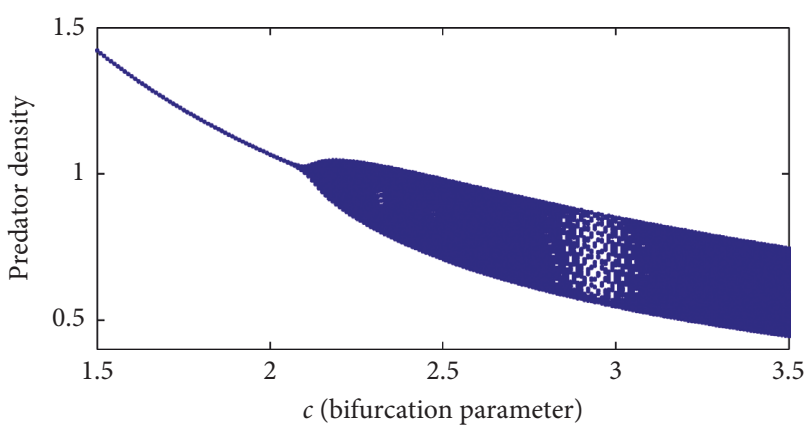

(b)

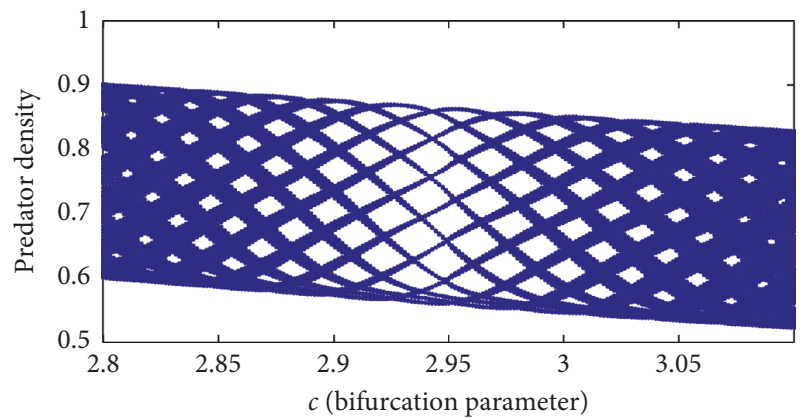

(d)

FIgURE 6: Orbit diagram of species for parameter $c$ and local amplification.

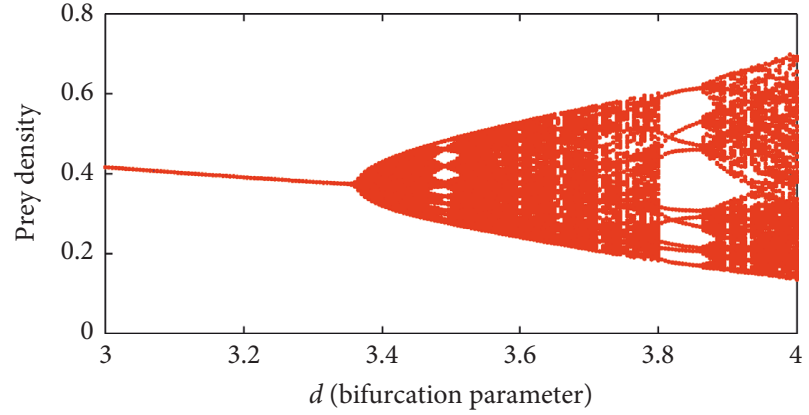

(a)

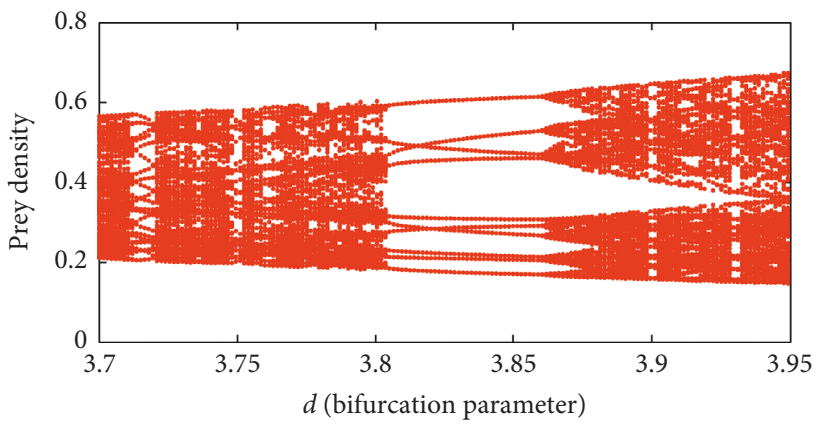

(c)

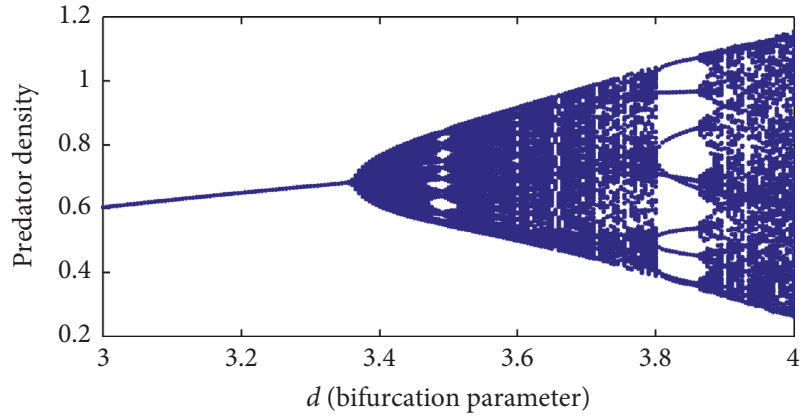

(b)

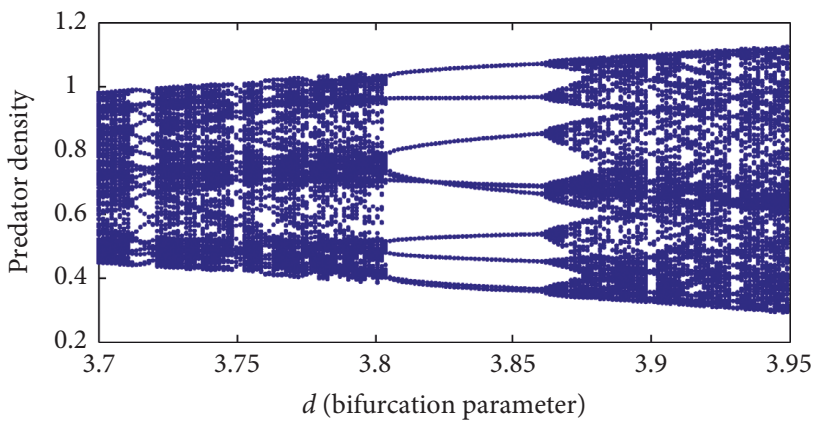

(d)

FIgURE 7: Orbit diagram of species for parameter $d$ and local amplification.

Period-10 orbit for $b=0.05$. Period-68 orbit for $b=0.06$. Attracting invariant cycle for $b=0.065,0.10,0.15$, and 0.152 . Fixed point for $b=0.153$ and 0.160 .
The orbit diagram of species for $a$, which is a scaling parameter of the intrinsic per capita growth rate of prey population. Figure 4 shows that the system trajectory evolves 


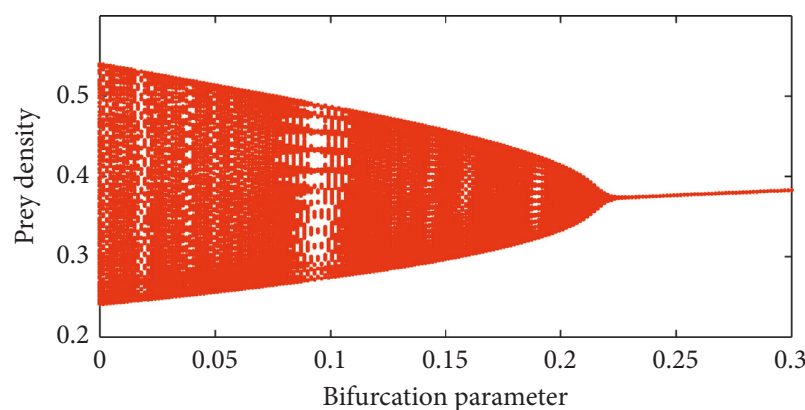

(a)

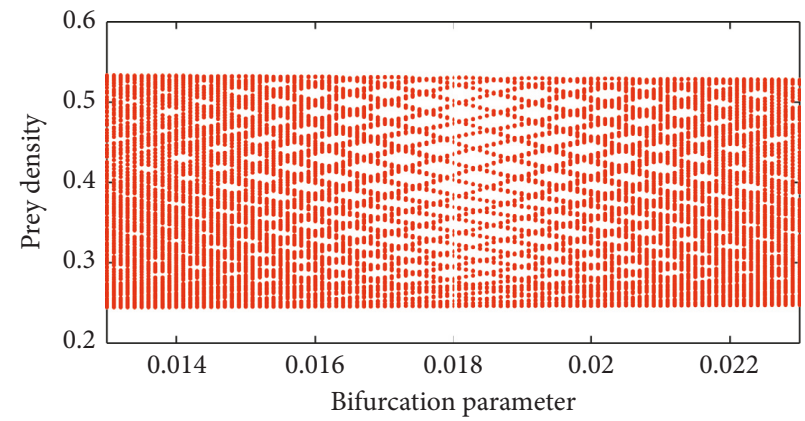

(c)

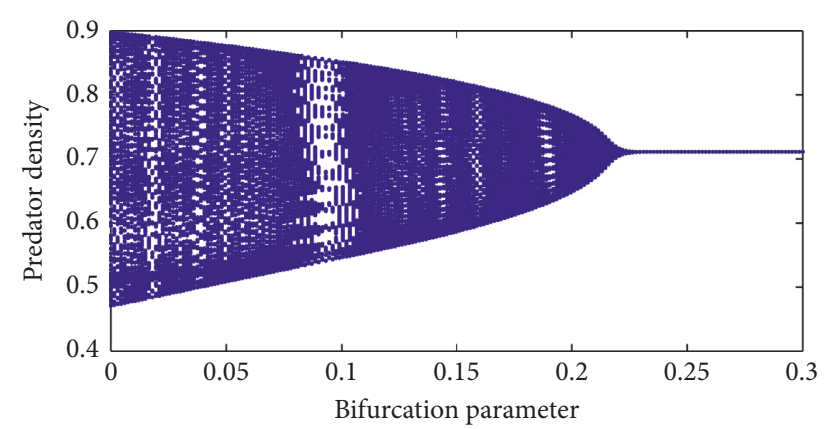

(b)

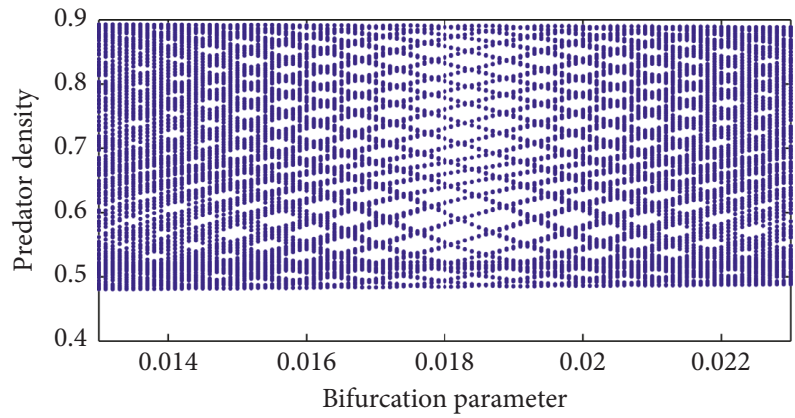

(d)

FIgURE 8: Orbit diagram of species for parameter $\alpha$ and local amplification.

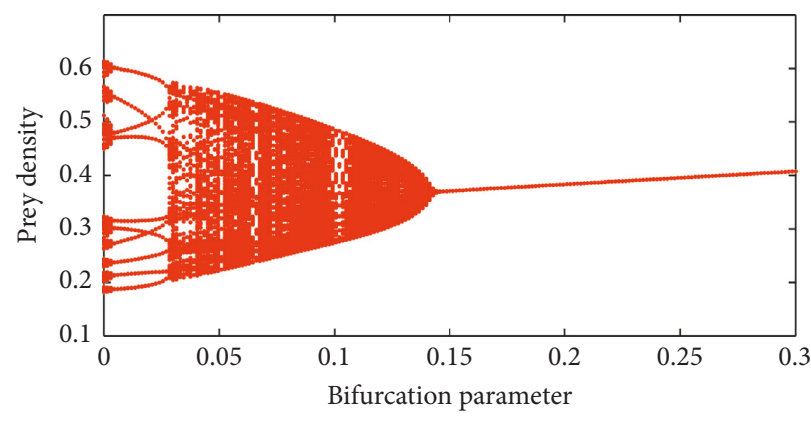

(a)

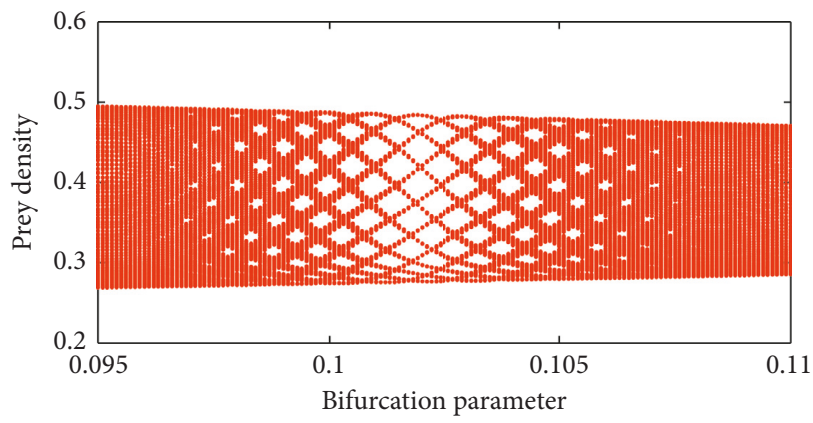

(c)

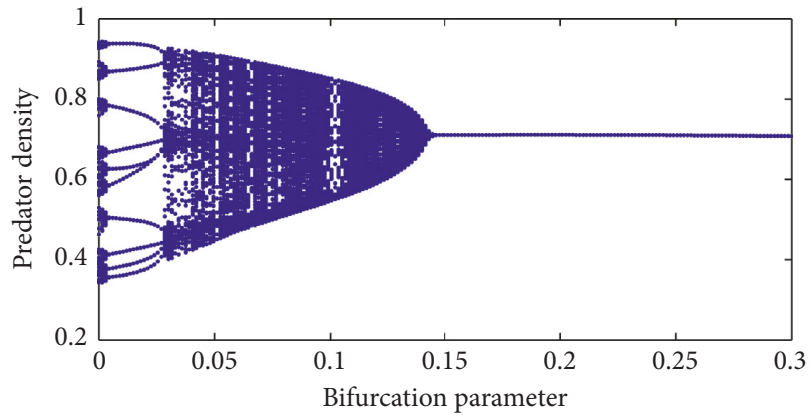

(b)

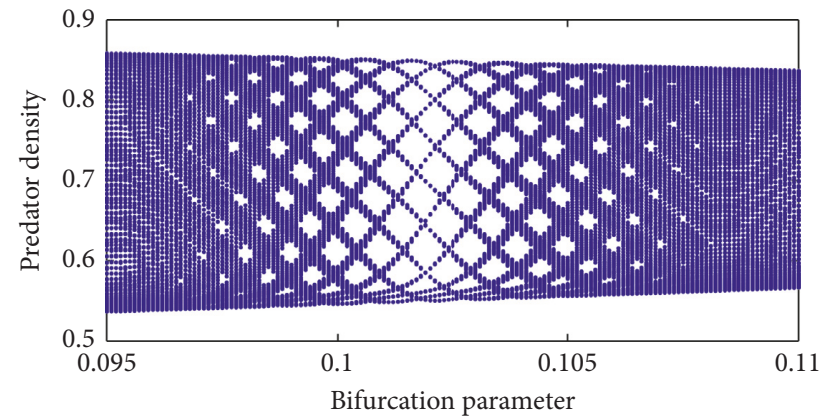

(d)

FIgURE 9: Orbit diagram of species for parameter $\beta$ and local amplification.

from a fixed point to NSB and finally into a chaotic attractor. There is one visible periodic windows in the bifurcation diagram for both prey and predator. We also give local magnification of the corresponding bifurcation figure.
Figure 10 is the corresponding Lyapunov exponents of Figure 4.

Figure 5 shows the orbit diagram of prey and predator population for the refuge parameter $(b)$ with other fixed 


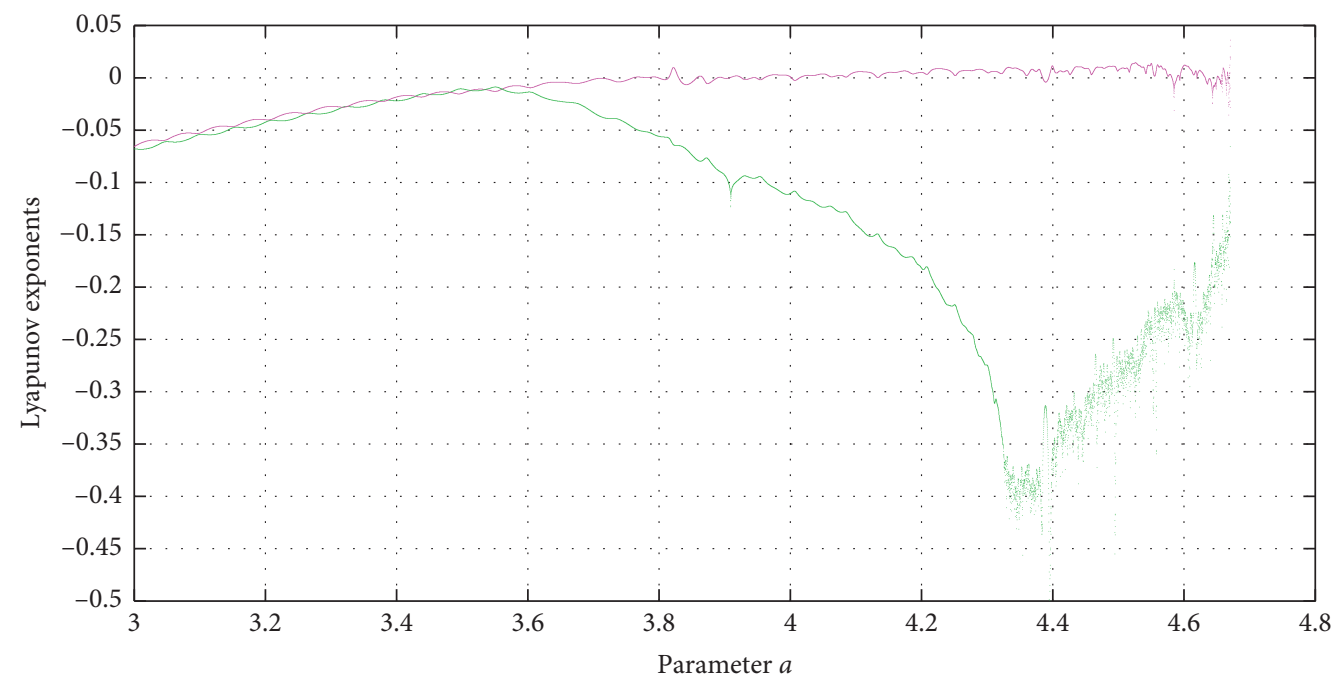

FIgURE 10: Lyapunov exponents of the system for $a$.

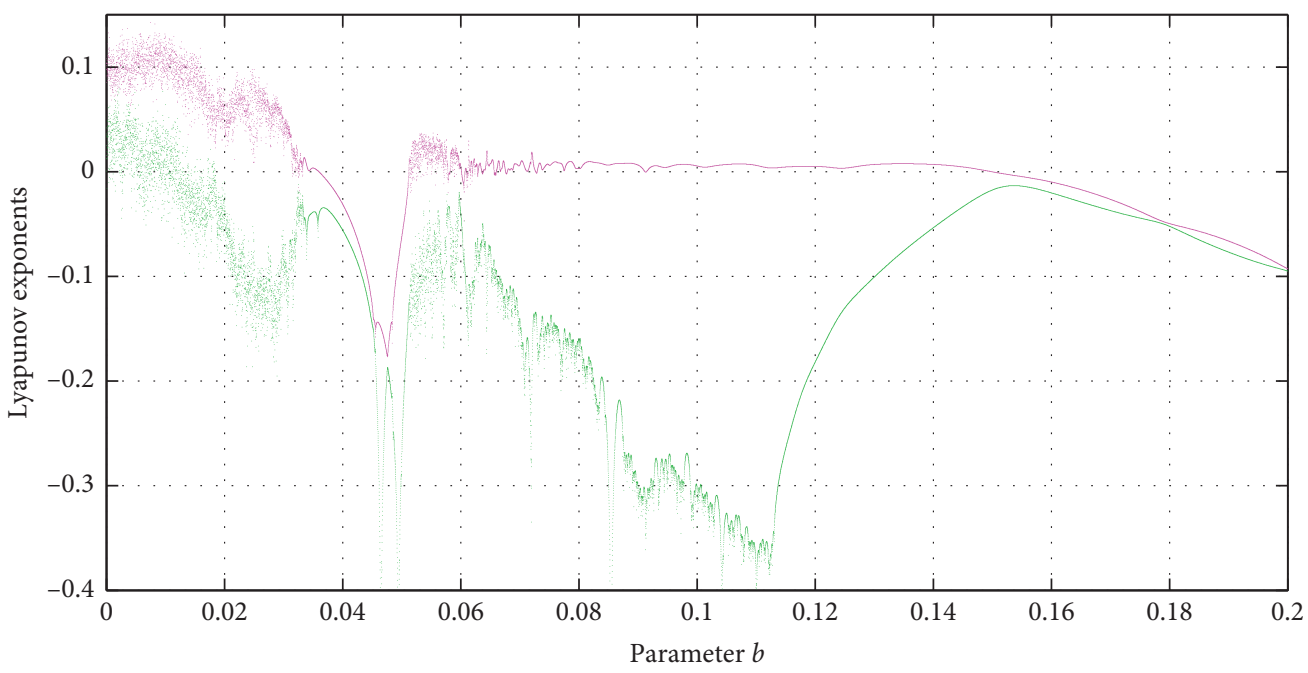

Figure 11: Lyapunov exponents of the system for $b$.

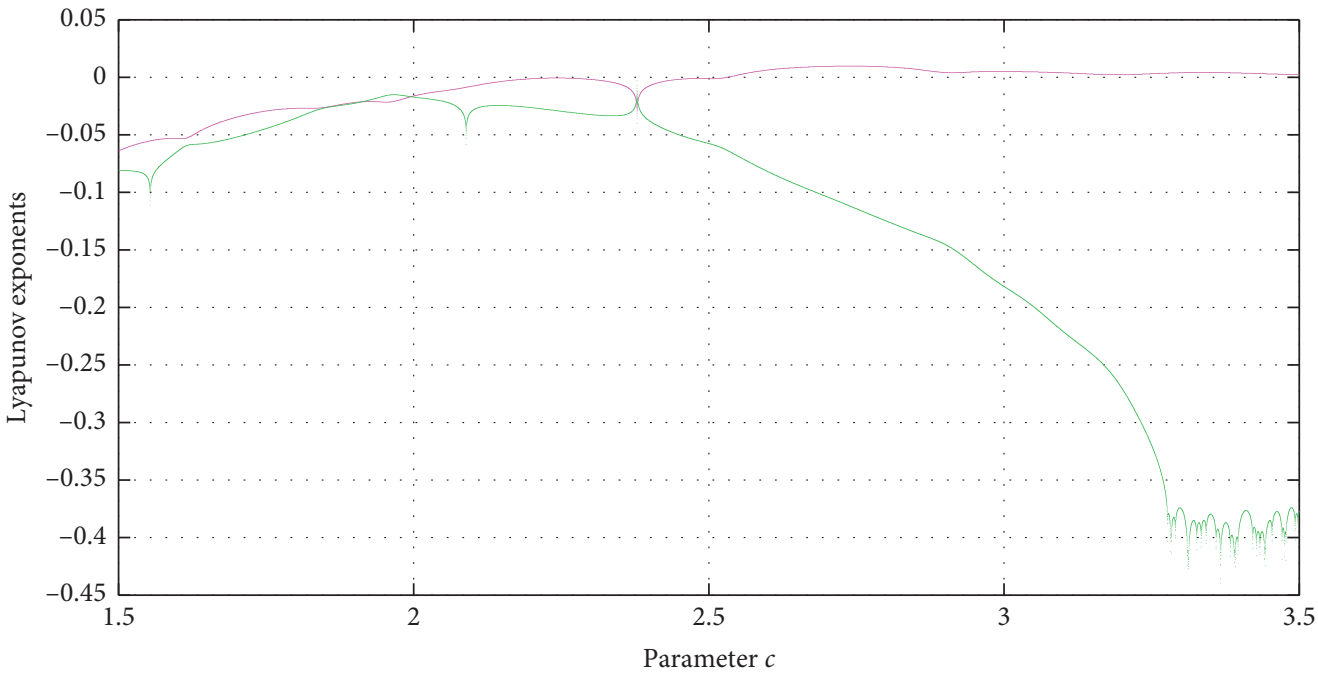

FIgURE 12: Lyapunov exponents of the system for $c$. 


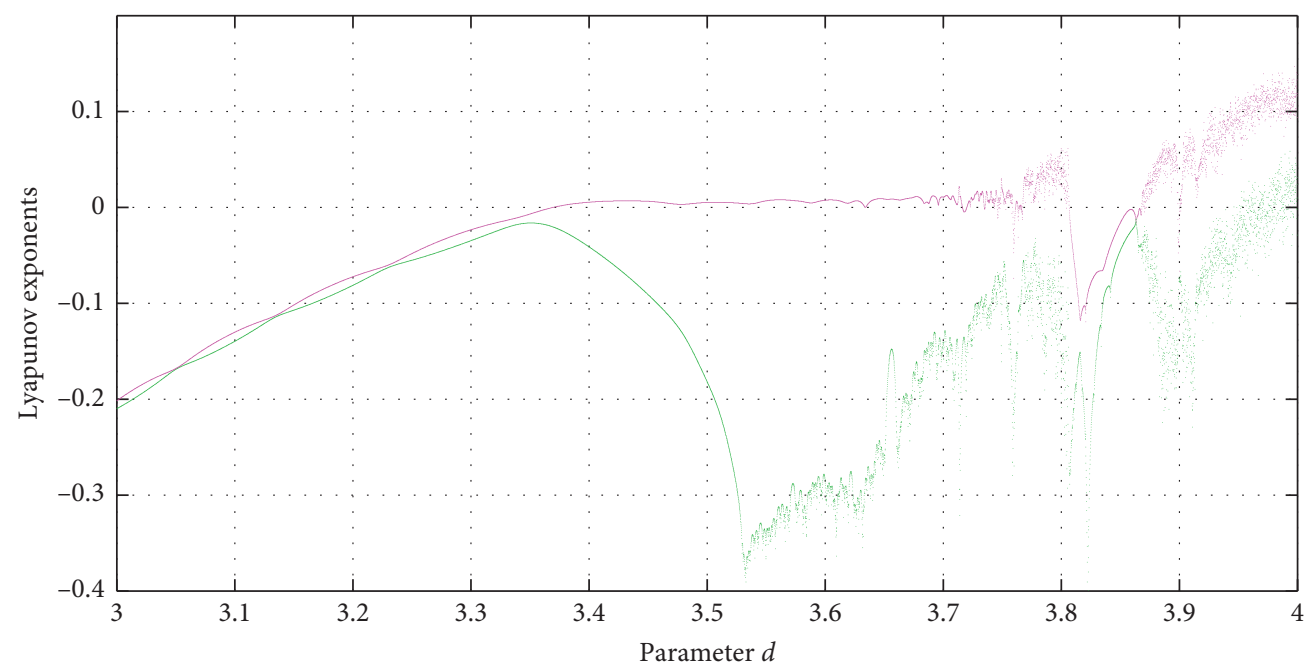

FIgURE 13: Lyapunov exponents of the system for $d$.

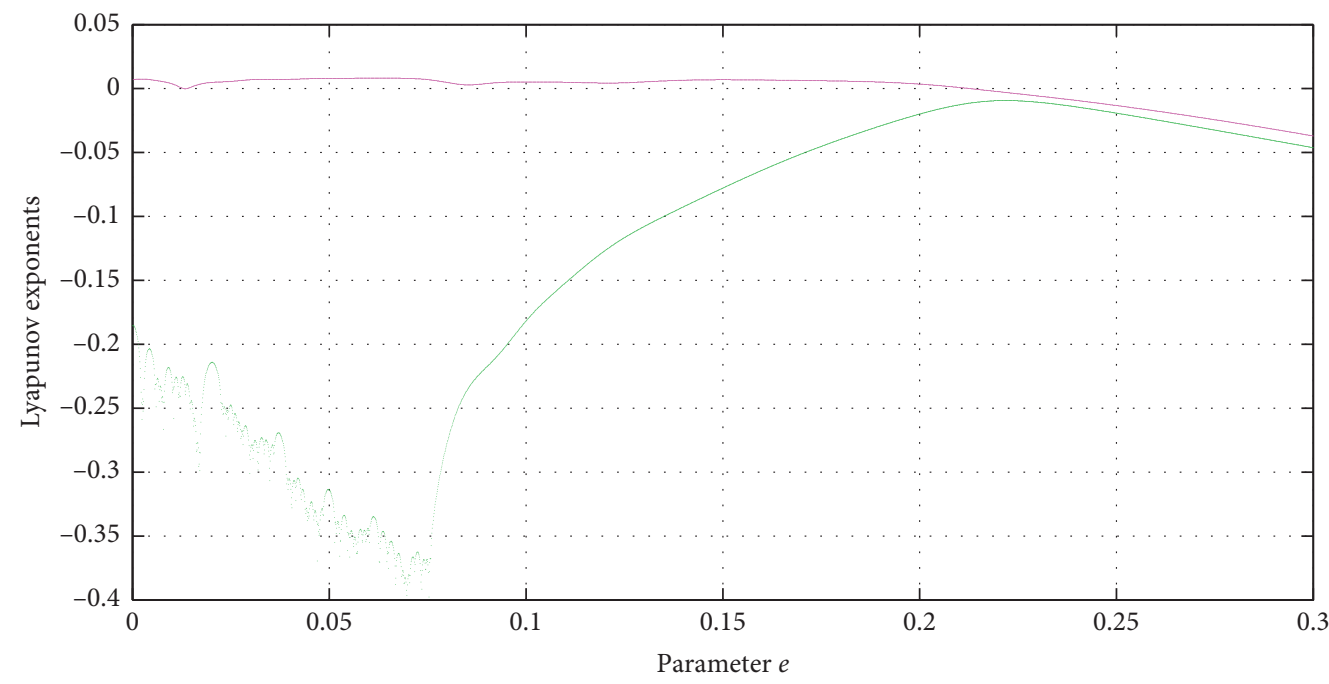

FIGURE 14: Lyapunov exponents of the system for $\alpha$.

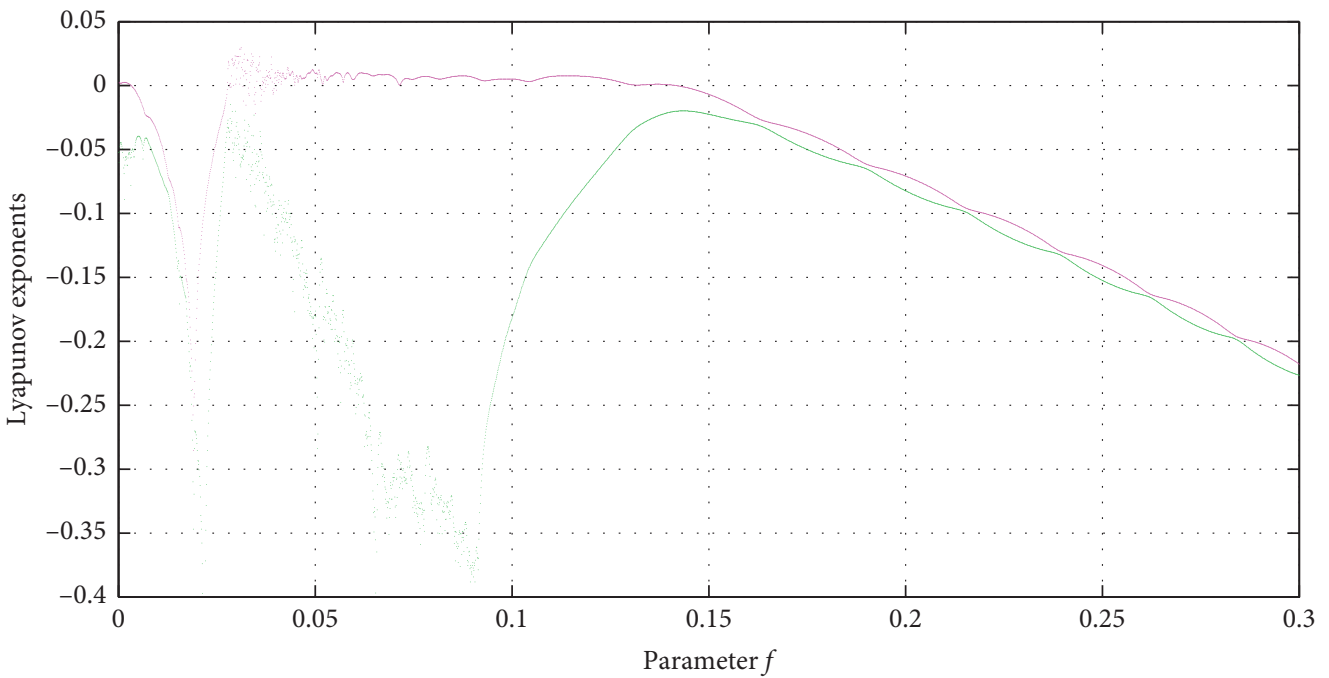

FIGURE 15: Lyapunov exponents of the system for $\beta$. 


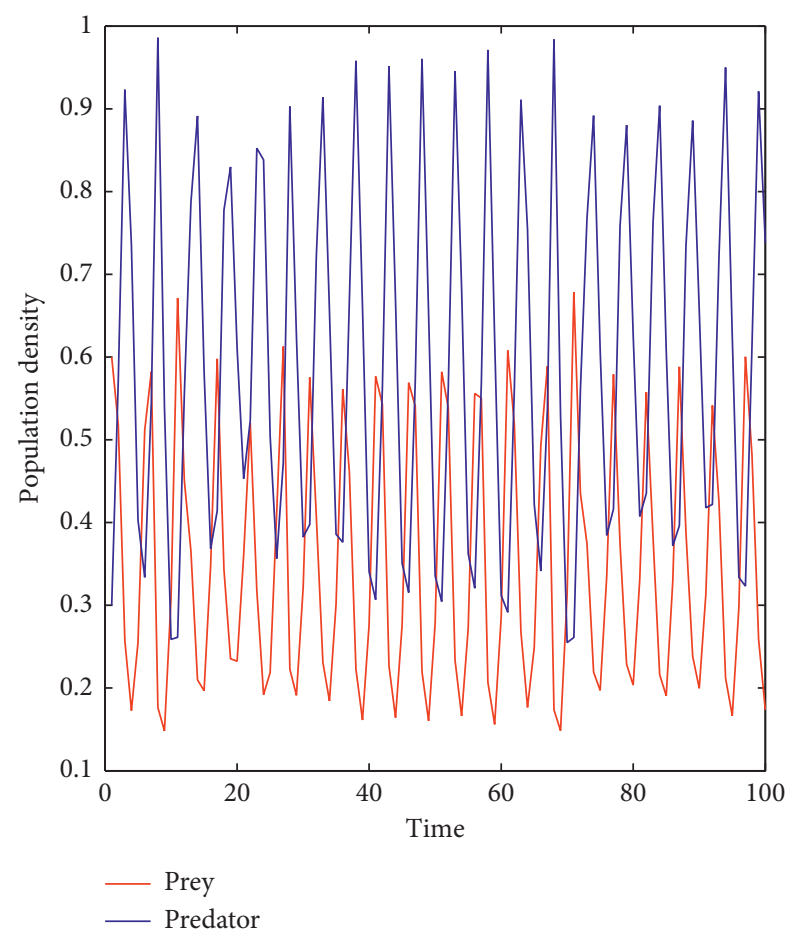

(a)

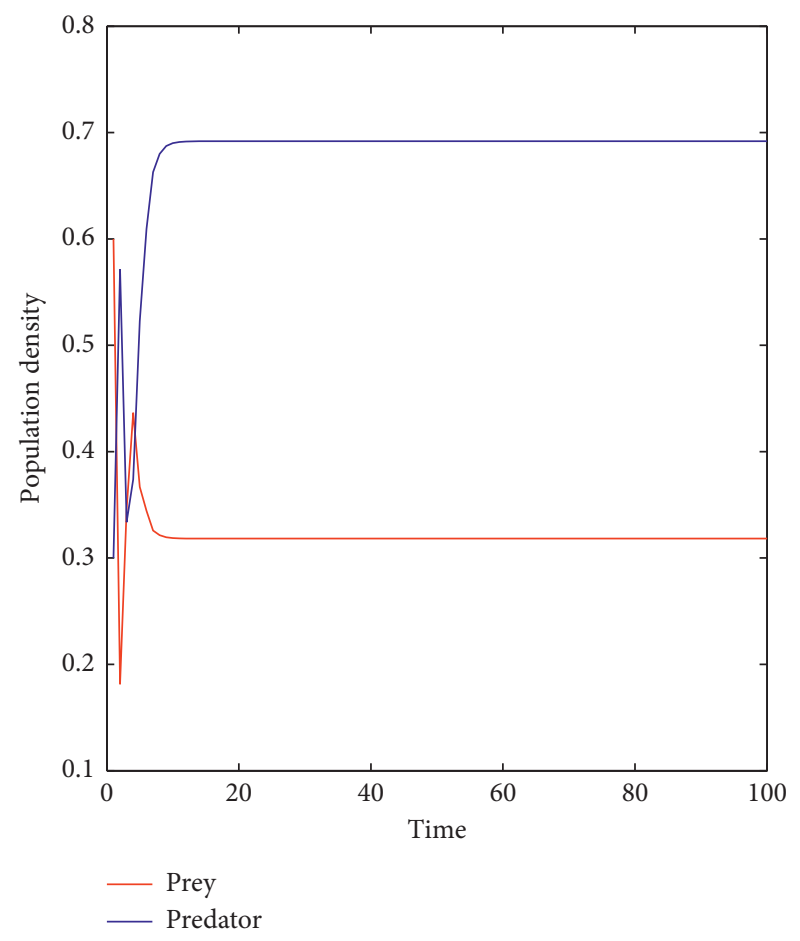

(b)

FIGURE 16: Chaos control.

parameter values. The system trajectory evolves into a periodic orbit from the chaotic attractor and finally into a fixed point. There is only one obvious periodic window in all the bifurcation process for both prey and predator. We also give local magnification of the corresponding bifurcation figure. Figure 11 is the corresponding Lyapunov exponent of Figure 5.

Figure 6 shows the orbit diagram of the population for the parameter $(c)$ of per capita consumption rate of predators. The system trajectory evolves from a fixed point to NSB and finally into a chaotic attractor. We also give local magnification of the corresponding bifurcation figure. Figure 12 is the corresponding Lyapunov exponent of Figure 6.

Figure 7 shows the orbit diagram of species for the parameter $(d)$ of efficiency with which predators convert the consumed prey into a new predator. The system trajectory evolves from a fixed point to NSB and finally into a chaotic attractor. There is one big visible periodic window in the bifurcation diagram for both prey and predator. We also give local magnification of the corresponding bifurcation figure. Figure 13 is the corresponding Lyapunov exponent of Figure 7.

Figure 8 shows the orbit diagram of prey and predator population for the parameter $\alpha$ with other fixed parameter values. The system trajectory evolves into a fixed point from the periodic orbit. We also give local magnification of the corresponding bifurcation figure. Figure 14 is the corresponding Lyapunov exponent of Figure 8.

Figure 9 shows the orbit diagram of prey and predator population for the parameter $\beta$ with other fixed parameter values. The system trajectory evolves into a periodic orbit from the chaotic attractor and finally into a fixed point. There is only one obvious periodic window in all the bifurcation process for both prey and predator. We also give local magnification of the corresponding bifurcation figure. Figure 15 is the corresponding Lyapunov exponent of Figure 9.

7.1. Chaos Control. We observe chaotic behavior of preypredator, as shown in Figure 16(a). In this case fixed point $(0.3183,0.6919)$ is unstable. In the feedback control method for feedback gain $q_{1}=0.5$ and $q_{2}=-0.5$, we observe the fixed point $(0.3183,0.6919)$ is stable, as shown in Figure 16(b).

7.2. Fractal Dimension. The fractal dimension is defined by using Lyapunov exponents as follows.

Let $f$ be a map on $R^{m}$. Consider an orbit with Lyapunov exponents $h_{1} \geq h_{2} \geq \ldots \geq h_{m}$, and let $p$ denote the largest integer such that $\sum_{i=1}^{i=1} h_{i} \geq 0$.

Fractal dimension $d_{L}= \begin{cases}0, & \text { if no such } p \text { exist, } \\ p+\frac{\sum_{i=1}^{i=p} h_{i}}{\left|h_{p+1}\right|}, & \text { if } p<m, \\ m, & \text { if } p=m .\end{cases}$

Fractal dimension and Lyapunov exponents for different values of $b$ for the value of the parameters as $a=4.2, c=3.0$, $d=3.5, \alpha=0.1$, and $\beta=0.1$ with initial population $(0.6,0.3)$ are presented in Table 1. 
TABLE 1: Fractal dimension and Lyapunov exponents for different values of $b$.

\begin{tabular}{lcc}
\hline Value of $b$ & Lyapunov exponents & Fractal dimension \\
\hline$b=0.0$ & $h_{1}=0.1295$ & 2 \\
& $h_{2}=0.0251$ & \\
\hline$b=0.01$ & $h_{1}=0.0956$ & 2 \\
& $h_{2}=0.0381$ & \\
\hline$b=0.02$ & $h_{1}=0.0538$ & 2.2036 \\
& $h_{2}=-0.0447$ & \\
$b=0.03$ & $h_{1}=0.0383$ & 1.3865 \\
& $h_{2}=-0.0991$ & \\
\hline
\end{tabular}

\section{Conclusion}

In this paper, we have studied the effects of refuges on a predator-prey interaction, by using the analytical and graphical approach. The refuges are considered as prey refuge proportional to prey density. We evaluate the effects with regard to the local stability of the interior equilibrium point and the long-term dynamics of the interacting populations. The results show that the effects of refuges can stabilize the interior equilibrium point of the proposed preypredator discrete-time domain model. We have studied bifurcations in a discrete predator-prey model with refuge. Furthermore, it is also shown that the model exhibits various bifurcations of codimension 1, including period-doubling bifurcation, and Neimark-Sacker bifurcation as the values of parameters vary. Numerical simulations and graphical presentation show the rich dynamics in bifurcation and chaotic nature of the system model.

\section{Data Availability}

No data were used to support this study.

\section{Conflicts of Interest}

The authors declare that there are no conflicts of interest regarding the publication of this paper.

\section{References}

[1] A. A. Berryman, "The orgins and evolution of predator-prey theory," Ecology, vol. 73, no. 5, pp. 1530-1535, 1992.

[2] A. R. Hausrath, "Analysis of a model predator-prey system with refuges," Journal of Mathematical Analysis and Applications, vol. 181, no. 2, pp. 531-545, 1994.

[3] M. E. Hochberg and R. D. Holt, "Refuge evolution and the population dynamics of coupled host-parasitoid associations," Evolutionary Ecology, vol. 9, no. 6, pp. 633-661, 1995.

[4] J. Maynard Smith, Models in Ecology, Cambridge University Press, Cambridge, UK, 1974.

[5] J. Guckenheimer and P. Holmes, Nonlinear Oscillations, Dynamical Systems and Bifurcations of Vector Fields, Springer-Verlag, Berlin, Germany, 1983.

[6] G. D. Ruxton, "Short term refuge use and stability of predatorprey models," Theoretical Population Biology, vol. 47, no. 1, pp. 1-17, 1995.
[7] T. W. Anderson, "Predator responses, prey refuges, and density-dependent mortality of a marine fish," Ecology, vol. 82, no. 1, pp. 245-257, 2001.

[8] E. González-Olivares and R. Ramos-Jiliberto, "Dynamic consequences of prey refuges in a simple model system: more prey, fewer predators and enhanced stability," Ecological Modelling, vol. 166, no. 1-2, pp. 135-146, 2003.

[9] Y. Huang, F. Chen, and L. Zhong, "Stability analysis of a preypredator model with holling type III response function incorporating a prey refuge," Applied Mathematics and Computation, vol. 182, no. 1, pp. 672-683, 2006.

[10] S. Magalhães, P. C. J. van Rijn, M. Montserrat, A. Pallini, and M. W. Sabelis, "Population dynamics of thrips prey and their mite predators in a refuge," Oecologia, vol. 150, no. 4, pp. 557-568, 2007.

[11] V. H. W. Rudolf and J. Armstrong, "Emergent impacts of cannibalism and size refuges in prey on intraguild predation systems," Oecologia, vol. 157, no. 4, pp. 675-686, 2008.

[12] Z. Ma, W. Li, Y. Zhao, W. Wang, H. Zhang, and Z. Li, "Effects of prey refuges on a predator-prey model with a class of functional responses: the role of refuges," Mathematical Biosciences, vol. 218, no. 2, pp. 73-79, 2009.

[13] F. Chen, L. Chen, and X. Xie, "On a Leslie-Gower predatorprey model incorporating a prey refuge," Nonlinear Analysis: Real World Applications, vol. 10, no. 5, pp. 2905-2908, 2009.

[14] R. Cressman and J. Garay, "A predator-prey refuge system: evolutionary stability in ecological systems," Theoretical Population Biology, vol. 76, no. 4, pp. 248-257, 2009.

[15] Y. Tao, X. Wang, and X. Song, "Effect of prey refuge on a harvested predator-prey model with generalized functional response," Communications in Nonlinear Science and $\mathrm{Nu}$ merical Simulation, vol. 16, no. 2, pp. 1052-1059, 2011.

[16] Y. Wang and J. Wang, "Influence of prey refuge on predatorprey dynamics," Nonlinear Dynamics, vol. 67, no. 1, pp. 191-201, 2012.

[17] S. Sarwardi, P. K. Mandal, and S. Ray, "Analysis of a competitive prey-predator system with a prey refuge," Biosystems, vol. 110, no. 3, pp. 133-148, 2012.

[18] J. Zhao, M. Zhao, and H. Yu, "Complex dynamical behavior of a predator-prey system with group defense," Mathematical Problems in Engineering, vol. 2013, Article ID 910349, 8 pages, 2013.

[19] Z. Ma, S. Wang, W. Li, and Z. Li, "The effect of prey refuge in a patchy predator-prey system," Mathematical Biosciences, vol. 243, no. 1, pp. 126-130, 2013.

[20] D. Pal and G. S. Mahapatra, "A bioeconomic modeling of twoprey and one-predator fishery model with optimal harvesting policy through hybridization approach," Applied Mathematics and Computation, vol. 242, pp. 748-763, 2014.

[21] M. Haque, M. S. Rahman, E. Venturino, and B.-L. Li, "Effect of a functional response-dependent prey refuge in a predatorprey model," Ecological Complexity, vol. 20, pp. 248-256, 2014.

[22] R. N. Fan, "A predator-prey model incorporating prey refuge and Allee effect," Applied Mechanics and Materials, vol. 713-715, pp. 1534-1539, 2015.

[23] D. Jana, R. Agrawal, and R. K. Upadhyay, "Dynamics of generalist predator in a stochastic environment: effect of delayed growth and prey refuge," Applied Mathematics and Computation, vol. 268, pp. 1072-1094, 2015.

[24] G. S. Mahapatra and P. Santra, "Prey-predator model for optimal harvesting with functional response incorporating prey refuge," International Journal of Biomathematics, vol. 9, no. 1, Article ID 1650014, 2016. 
[25] D. Pal and G. S. Mahapatra, "Effect of toxic substance on delayed competitive allelopathic phytoplankton system with varying parameters through stability and bifurcation analysis," Chaos, Solitons \& Fractals, vol. 87, pp. 109-124, 2016.

[26] P. Santra, G. S. Mahapatra, and D. Pal, "Prey-predator nonlinear harvesting model with functional response incorporating prey refuge," International Journal of Dynamics and Control, vol. 4, no. 3, pp. 293-302, 2016.

[27] J. Ghosh, B. Sahoo, and S. Poria, "Prey-predator dynamics with prey refuge providing additional food to predator," Chaos, Solitons \& Fractals, vol. 96, pp. 110-119, 2017.

[28] Z. Ma, S. Wang, T. Wang, and H. Tang, "Stability analysis of prey-predator system with holling type functional response and prey refuge," Advances in Difference Equations, vol. 2017, no. 1, p. 243, 2017.

[29] D. Pal, P. Santra, and G. S. Mahapatra, "Dynamical behavior of three species predator-prey system with mutual support between non refuge prey," Ecological Genetics and Genomics, vol. 3-5, pp. 1-6, 2017.

[30] Q. Wang, Z. Liu, X. Zhang, R. A. Cheke, and A. Cheke, "Incorporating prey refuge into a predator-prey system with imprecise parameter estimates," Computational and Applied Mathematics, vol. 36, no. 2, pp. 1067-1084, 2017.

[31] S. H. Levine, "Discrete time modeling of ecosystems with applications in environmental enrichment," Mathematical Biosciences, vol. 24, no. 3-4, pp. 307-317, 1975.

[32] M. G. Neubert and M. Kot, "The subcritical collapse of predator populations in discrete-time predator-prey models," Mathematical Biosciences, vol. 110, no. 1, pp. 45-66, 1992.

[33] J.-C. Huang, "Bifurcations and chaos in a discrete predatorprey system with holling type-IV functional response," Acta Mathematicae Applicatae Sinica, English Series, vol. 21, no. 1, pp. 157-176, 2005.

[34] X. Liu and D. Xiao, "Bifurcations in a discrete time LotkaVolterra predator-prey system," Discrete and Continuous Dynamical Systems-Series B, vol. 6, no. 3, pp. 559-572, 2006.

[35] X. Liu and D. Xiao, "Complex dynamic behaviors of a discrete-time predator-prey system," Chaos, Solitons \& Fractals, vol. 32, no. 1, pp. 80-94, 2007.

[36] C. Çelik and O. Duman, "Allee effect in a discrete-time predator-prey system," Chaos, Solitons \& Fractals, vol. 40, no. 4, pp. 1956-1962, 2009.

[37] X. Liu, "A note on the existence of periodic solutions in discrete predator-prey models," Applied Mathematical Modelling, vol. 34, no. 9, pp. 2477-2483, 2010.

[38] Z. Hu, Z. Teng, and L. Zhang, "Stability and bifurcation analysis of a discrete predator-prey model with nonmonotonic functional response," Nonlinear Analysis: Real World Applications, vol. 12, no. 4, pp. 2356-2377, 2011.

[39] Z. He and X. Lai, "Bifurcation and chaotic behavior of a discrete-time predator-prey system," Nonlinear Analysis: Real World Applications, vol. 12, no. 1, pp. 403-417, 2011.

[40] A.-E. A. Elsadany, "Dynamical complexities in a discrete-time food chain," Computational Ecology and Software, vol. 2, no. 2, pp. 124-139, 2012.

[41] Q. Din, "Dynamics of a discrete Lotka-Volterra model," Advances in Difference Equations, vol. 2013, no. 1, pp. 1-13, 2013.

[42] M. Zhao and Y. Du, "Stability of a discrete-time predator-prey system with allee effect," Nonlinear Analysis and Differential Equations, vol. 4, no. 5, pp. 225-233, 2016.

[43] M. Gámez, I. López, C. Rodríguez, Z. Varga, and J. Garay, "Ecological monitoring in a discrete-time prey-predator model," Journal of Theoretical Biology, vol. 429, no. 21, pp. 52-60, 2017.
[44] H. Baek, "Complex dynamics of a discrete-time predator-prey system with ivlev functional response," Mathematical Problems in Engineering, vol. 2018, Article ID 8635937, 15 pages, 2018.

[45] J. Huang, S. Liu, S. Ruan, and D. Xiao, "Bifurcations in a discrete predator-prey model with nonmonotonic functional response," Journal of Mathematical Analysis and Applications, vol. 464, no. 1, pp. 201-230, 2018.

[46] R. K. Upadhyay and R. K. Naji, "Dynamics of a three species food chain model with Crowley-Martin type functional response," Chaos, Solitons \& Fractals, vol. 42, no. 3, pp. 1337-1346, 2009.

[47] J. Zhou, "Qualitative analysis of a modified Leslie-Gower predator-prey model with Crowley-Martin functional responses," Communications on Pure and Applied Analysis, vol. 14, no. 3, pp. 1127-1145, 2015.

[48] J. P. Tripathi, S. Tyagi, and S. Abbas, "Global analysis of a delayed density dependent predator-prey model with Crowley-Martin functional response," Communications in Nonlinear Science and Numerical Simulation, vol. 30, p. 4, 2016.

[49] X. Li, X. Lin, and J. Liu, "Existence and global attractivity of positive periodic solutions for a predator-prey model with Crowley-Martin functional response," Electronic Journal of Differential Equations, vol. 191, pp. 1-17, 2018. 


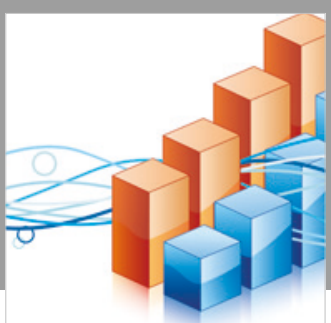

Advances in

Operations Research

\section{-n-m}
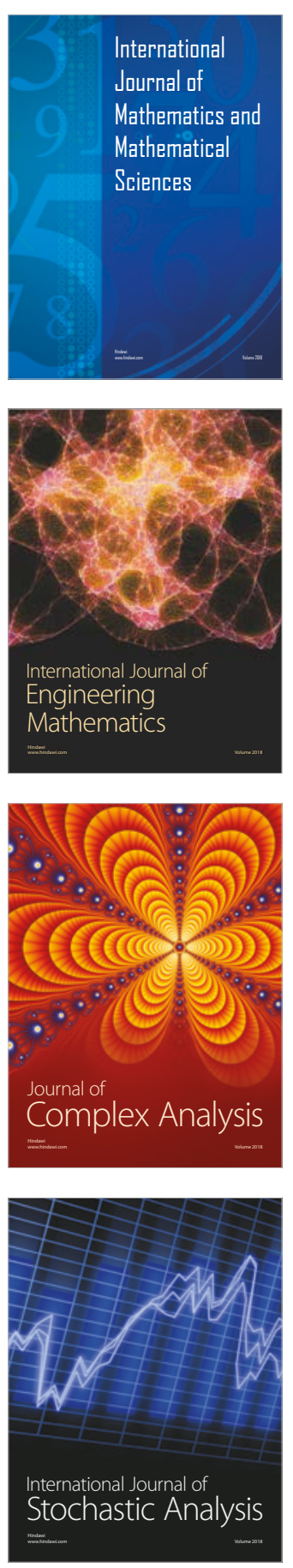
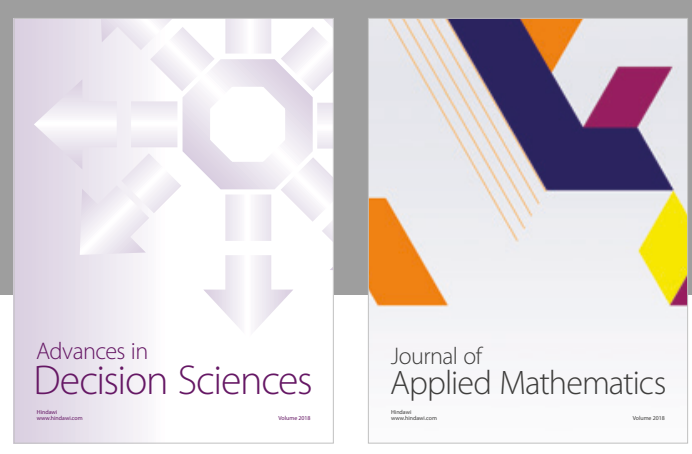

Journal of

Applied Mathematics
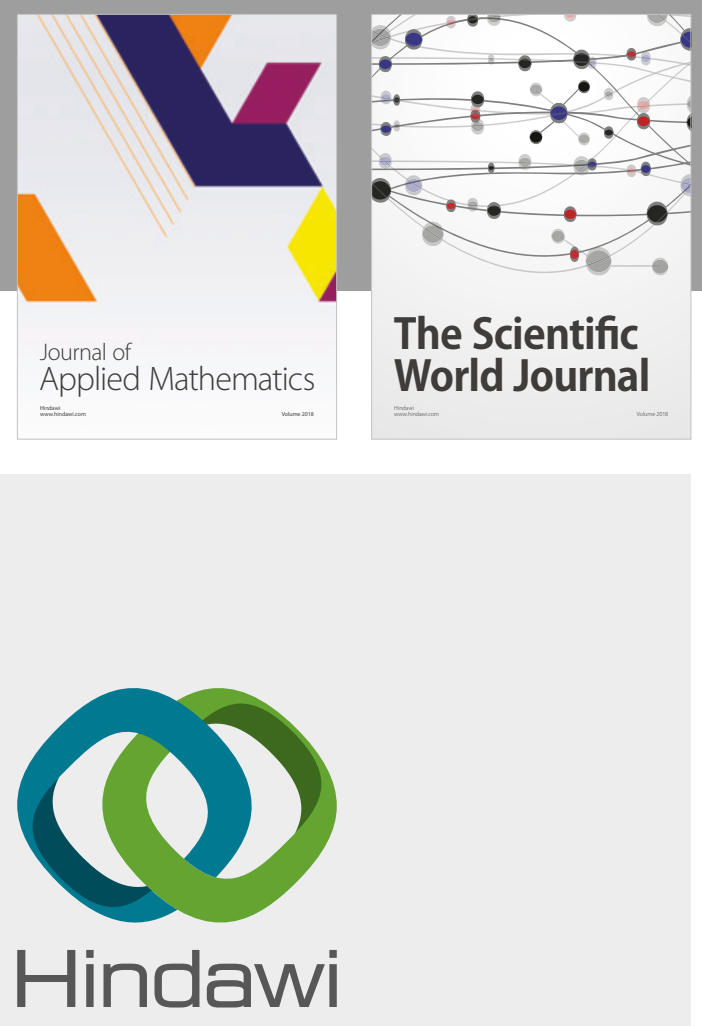

Submit your manuscripts at

www.hindawi.com

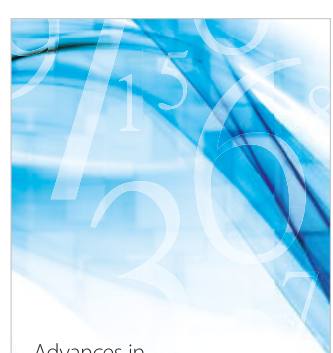

Advances in
Numerical Analysis
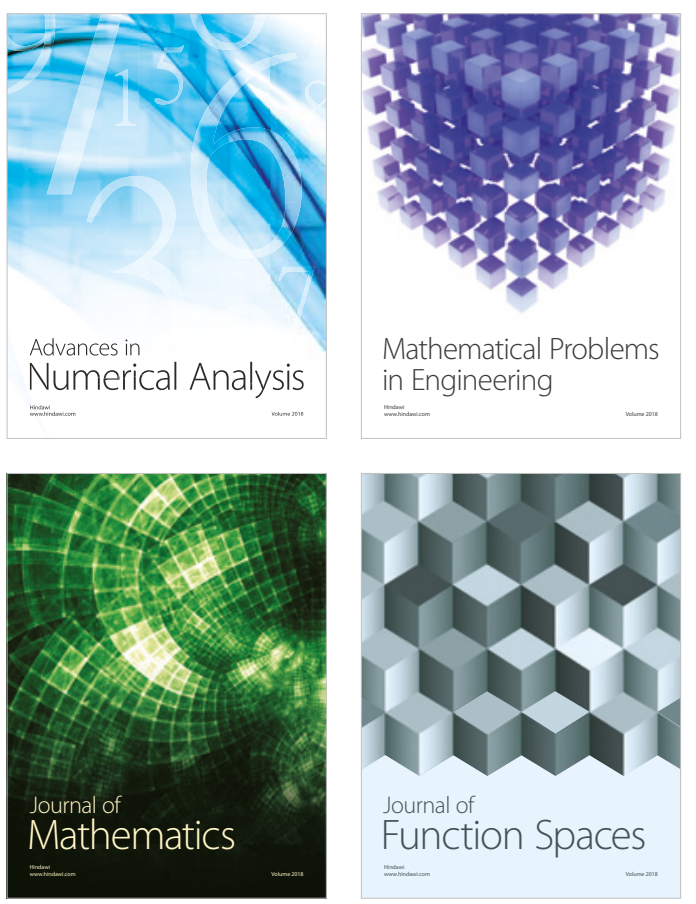

Mathematical Problems in Engineering

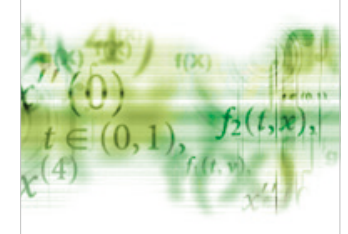

International Journal of

Differential Equations

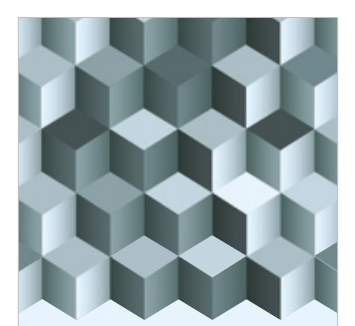

Journal of

Function Spaces

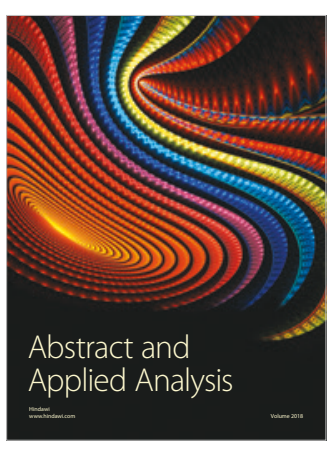

The Scientific

World Journal

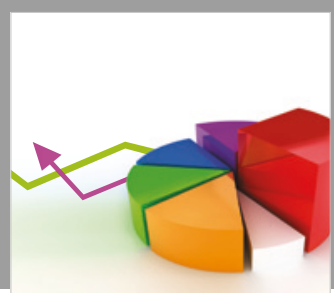

Journal of

Probability and Statistics
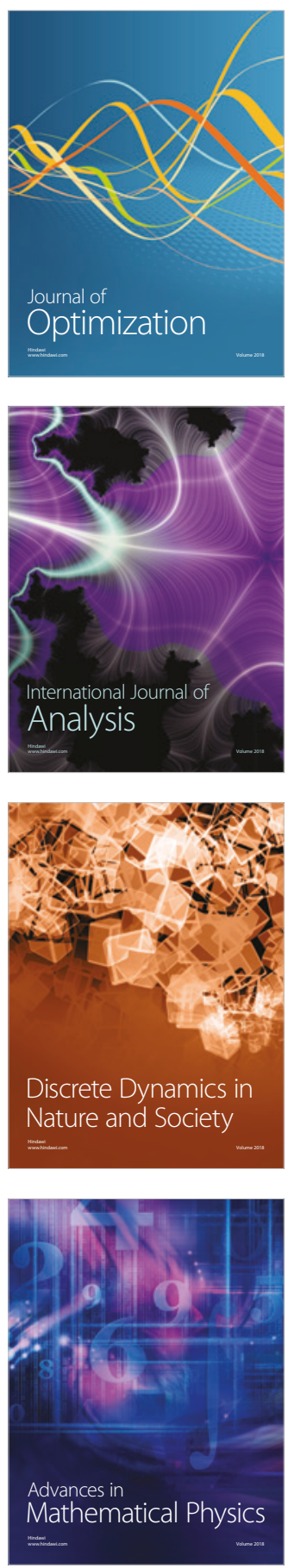